\title{
Underwater Broadband Source Localization Based on Modal Filtering and Features Extraction
}

\author{
Maciej Lopatka, ${ }^{1}$ Grégoire Le Touzé, ${ }^{1}$ Barbara Nicolas, ${ }^{1}$ Xavier Cristol, ${ }^{2}$ \\ Jérôme I. Mars, ${ }^{1}$ and Dominique Fattaccioli ${ }^{3}$ \\ ${ }^{1}$ GIPSA-Lab, Department of Image Signal, 961 rue de la Houille Blanche, 38402 St Martin d'Heres, France \\ ${ }^{2}$ THALES Underwater Systems S.A.S., 525 Route des Dolines BP 157, 06903 Sophia-Antipolis Cedex, France \\ ${ }^{3}$ CTSN-DGA Centre Technique des Systmes Navals, avenue de la Tour Royale BP 40915, 83050 Toulon Cedex, France
}

Correspondence should be addressed to Maciej Lopatka, maciej.lopatka@me.com

Received 7 July 2009; Accepted 11 January 2010

Academic Editor: Gary Wood

Copyright (C) 2010 Maciej Lopatka et al. This is an open access article distributed under the Creative Commons Attribution License, which permits unrestricted use, distribution, and reproduction in any medium, provided the original work is properly cited.

Passive source localization is a crucial issue in underwater acoustics. In this paper, we focus on shallow water environment (0 to $400 \mathrm{~m}$ ) and broadband Ultra-Low Frequency acoustic sources ( 1 to $100 \mathrm{~Hz}$ ). In this configuration and at a long range, the acoustic propagation can be described by normal mode theory. The propagating signal breaks up into a series of depth-dependent modes. These modes carry information about the source position. Mode excitation factors and mode phases analysis allow, respectively, localization in depth and distance. We propose two different approaches to achieve the localization: multidimensional approach (using a horizontal array of hydrophones) based on frequency-wavenumber transform ( $F-K$ method) and monodimensional approach (using a single hydrophone) based on adapted spectral representation ( $F T_{a}$ method). For both approaches, we propose first complete tools for modal filtering, and then depth and distance estimators. We show that adding mode sign and source spectrum informations improves considerably the localization performance in depth. The reference acoustic field needed for depth localization is simulated with the new realistic propagation modelMoctesuma. The feasibility of both approaches, $F-K$ and $F T_{a}$, are validated on data simulated in shallow water for different configurations. The performance of localization, in depth and distance, is very satisfactory.

\section{Introduction}

Passive source localization in shallow water has attracted much attention for many years in underwater acoustics. In this environment and for Ultra-Low Frequency waves ( 1 to $100 \mathrm{~Hz}$, denoted further ULF) classical beamforming techniques are inappropriate because they do not consider multipath propagation phenomena and ocean acoustic channel complexity. Indeed, ULF acoustic propagation in shallow water waveguides is classically based on normal mode theory [1]. ULF band is very attractive for detection, localization, and geoacoustical parameter estimation purposes, because propagating acoustic waves are almost not affected by absorption and thus can propagate at very long ranges. In this context, mainly two approaches are used: Matched-Field Processing (denoted MFP) $[2,3]$ and Matched-Mode Processing (denoted MMP) [4-6]. The com- parative study of both approaches is given in [7]. MatchedMode approach can be considered as MFP combined with modal decomposition. The main difference is that MFP operates in receiver space and MMP in mode space. Both methods require a reference acoustic field (replica field) to be compared, generally by correlation techniques (building and maximizing an objective function), with the real acoustic field recorded on receiver(s). Another alternative to perform source localization is to use time reversal [8] which can be seen as a broadband coherent MFP. Some experiments have been performed showing the feasibility of the method [9]. The main drawback of the method is that a numerical backpropagation has to be computed which needs a good knowledge of the environment. As MMP is less sensitive to environmental mismatches than MFP and Time Reversal methods, this technique is more interesting for practical applications, and thus is used in our approach to estimate 
the source depth. The access to modes not only allows estimation of mode excitation factors for depth localization, but also gives the possibility to analyze mode phase to extract information about the source distance. As a result, in this paper depth estimation is performed using MMP on the mode excitation factors and distance estimation is achieved by mode phase analysis.

Consequently, the main issue to perform underwater localization for ULF sources in shallow water is to develop signal processing methods to accomplish modal filtering. These methods should be based on physics of wave propagation in waveguides, to be adapted to signals propagating in shallow water environment. In this context, we propose two complementary techniques to localize broadband impulsive source in depth and distance. The first method based on frequency-wavenumber transform and denoted $F-K$ is a multidimensional approach based on array processing. The second method based on adapted Fourier transform and denoted $F T_{a}$ is a monodimensional technique used on a single hydrophone.

Traditionally, matched-mode localization was applied on vertical line arrays (VLAs), and mode excitation factors were extracted by a spatial integration of pressure field. As proposed in $[10,11]$, we record the signal (represented in the space: radial distance $r$ and time $t$ ) on a horizontal line array (HLA), as it is generally more practical in real applications (towing possibility, faster deployment, and stability). In this configuration, modes can be filtered in the frequencywavenumber plane $(f-k)$, which is a two-dimensional Fourier transform of radial distance-time section ( signal $r-t)$.

For the mono-dimensional approach, modes cannot be filtered by conventional modal filtering techniques. As modes have nonstationary properties, the only way to filter modes is to integrate modal time-frequency characteristics [12] into modal filtering. The main idea is to deform a signal in such way that nonlinearities in the time-frequency plane become linear (according to the frequency domain). Consequently, the signal becomes stationary and classical filtering tools can be used to filter modes. Hereby, modal filtering in mono-dimensional configuration is done in an adapted frequency domain (Pekeris Fourier transform). The classical and adapted frequency domains are related by the unitary equivalence formalism [13].

After a brief presentation of modal propagation theory, we give a short description of the simulator Moctesuma-2006, which is used for simulation of acoustic replica fields and acoustic parameter computation. Then, we present details about the experimental configuration. Next, we describe mode filtering methods in the mono- and multidimensional cases to finally present estimators used for depth and distance localization. Finally, results of distance and depth localization for mono and multidimensional method are presented on simulated data.

\section{Modal Propagation and Modes}

Acoustic propagation of Ultra-Low Frequency waves in shallow water waveguide can be modeled by normal mode theory. Propagating signal at long range is composed of dispersive modes. These modes are analyzed for depth (matched-mode processing) and distance (mode phase processing) localization.

To demonstrate very succinctly the idea of localization using modes, we introduce the simplest model of oceanic waveguide - the perfect waveguide. Even if this model is a simplification of real complex waveguides, it reflects the most important waveguide phenomena: modal decomposition of the propagated signal.

The perfect waveguide model is made of a homogeneous layer of water between perfectly reflecting boundaries (a pressure release surface and a rigid sea bottom). The water layer is characterized by depth $D$, velocity $V_{1}$, and density $\rho_{1}$. We consider an omnidirectional point source located at depth $z_{s}$ and at distance 0 radiating a signal $s(t)$. The acoustic pressure field $P(r, z, t)$ received at a reception point of coordinates $(r, z)$, where $r$ and $z$ are, respectively, horizontal distance and depth, can be expressed by

$$
P(r, z, t)=\int_{f} p(r, z, f) \cdot e^{-2 i \pi f t} d f
$$

where $t$ is the time, $f$ is the frequency, and $p(r, z, f)$ satisfies the general Helmholtz equation.

Using variable separation (in depth and in range) and boundaries conditions [1], the pressure $p$ at long range becomes a sum of modes

$$
p(r, z, f)=A \cdot S(f) \cdot \sum_{m=1}^{\infty} \psi_{m}\left(z_{s}\right) \psi_{m}\left(z_{r}\right) \frac{e^{-2 i \pi k_{r m} r}}{\sqrt{k_{r m} r}}
$$

with $A$ a constant, $S(f)$ the source spectrum, $\psi_{m}$ the modal function of mode $m$, and $k_{r m}$ the horizontal spatial frequency of mode $m$. The spatial frequency $k$ is defined by $k=$ $f / V_{1}$ and it corresponds to the classical wavenumber divided by $2 \pi$. In the following, for the sake of simplicity, we will call $k$ the wavenumber with its horizontal and vertical components $k_{r}$ and $k_{z}$ (instead of spatial frequency). The mode wavenumber spectrum is discrete and each mode is associated with an unique wavenumber. The amplitude $\psi_{m}\left(z_{s}\right)$ is a function of the source depth $z_{s}$ :

$$
\psi_{m}\left(z_{s}\right)=\sqrt{\frac{2}{D}} \sin \left(k_{z m} z_{s}\right)
$$

with $k_{z m}=(2 m-1) / 4 D$.

This short theoretical introduction of normal mode theory made on the example of perfect waveguide exposes the principle used for source depth estimation; mode amplitudes depend on source depth $z_{s}$ by the factor $\psi_{m}\left(z_{s}\right)$.

Then, let us demonstrate very shortly the principle of mode phase processing for distance estimation. Modes contain, besides depth information, also distance information about the source. This information is contained in mode phase. The phase of mode $m$ at frequency $f$ is defined by

$$
\begin{aligned}
\Phi_{m}(f)= & \frac{\pi}{4}+\phi_{s}(f)+2 \pi f t_{0} \\
& +\phi\left(\psi_{m}\left(z_{s}\right)\right)+\phi\left(\psi_{m}\left(z_{r}\right)\right)+k_{r m}(f) r,
\end{aligned}
$$




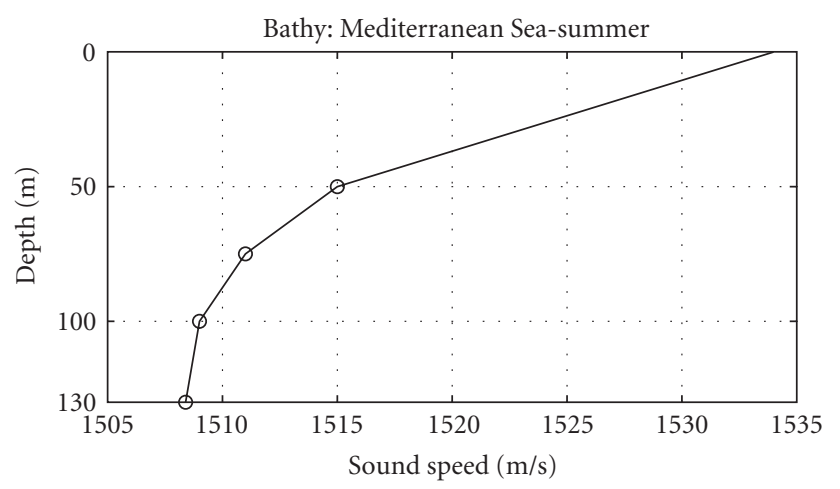

FIgURE 1: Sound speed profile of the water column for Mediterranean Sea in summer.

where

(i) $\phi_{s}(f)$ is the phase of the source at frequency $f$;

(ii) $2 \pi f t_{0}$ is a phase factor due to time delay $t_{0}$ of the recorded signal;

(iii) $\phi\left(\psi_{m}\left(z_{s}\right)\right)$ depends on the modal function sign at the source depth $z_{s}$; it is $\pi$ if $\psi_{m}\left(z_{s}\right)<0$ and 0 if $\psi_{m}\left(z_{s}\right) \geq$ 0 ;

(iv) $\phi\left(\psi_{m}\left(z_{r}\right)\right)$ depends on the modal function sign at the receiver depth $z_{r}$;

(v) $k_{r m}(f) r$ is a phase factor at frequency $f$ linked to the propagation distance $r$ between source and receiver.

As one can notice, modal decomposition is a very useful theory for acoustic propagation in oceanic waveguide. Indeed, MMP uses this decomposition to perform localization [14].

In this section we demonstrated that by having access to modes, and more precisely to their excitation factors and phases, it is possible to localize source in depth and in distance.

Moctesuma-2006. To perform the depth estimation using MMP, we need an acoustic model to generate replica fields. Several classical underwater acoustic propagation models exist in the literature and are used according to the seabed depth, the source range, and the frequency band. Models are based on different theories: ray theory, parabolic equation modeling, normal mode models, and spectral integral models [1]. Among the different models we choose the numerical model Moctesuma-2006-a realistic underwater acoustic propagation simulator developed by Thales Underwater Systems [15]. For the sake of simplicity Moctesuma2006 will be called further Moctesuma. This model, based on normal mode theory, simulates an underwater acoustic propagation for range-dependent environments. It is well adapted to transient broadband ULF signals for shallow and deep water environments. Moreover, we choose Moctesuma as it provides the acoustic parameters of the environment (wavenumbers) and the full acoustic field (time-series) [16].

The transmitted transient signal is first split into narrow subbands signals through a set of bandpass filters. Each subband is associated with a central frequency for which acoustic modes are fully computed. For each mode in each subband, propagation consists in delaying the original signal. The summation is performed in the time domain, so the signal causality is necessarily satisfied. Moctesuma considers different acoustic signal phenomena such as penetration, elasticity, multiple interactions inside multilayered sea bottoms and water. The time and space structure of waves is analyzed beyond simple wavefronts and Doppler effect (moving source and/or receiver).

A set of parameters is necessary to make a simulation with Moctesuma. The first parameter group concerns a description of the environment. As it is a range-dependent model, parameters are given for each environment sector. User has to provide following environmental parameters: sea state, temperature, sound speed profile, seabed type (or precise seabed structure). The second parameter group concerns the input signal and the experiment configuration (coordinates, depths, speeds and caps of the source and the antenna, antenna's length, and sensors' number).

In our analysis, we use Moctesuma to simulate the reference acoustic field (in the MMP) and to access the acoustic parameters of the environment such as horizontal wavenumbers, group velocities, and mode excitation factors signs.

\section{Experimental Configuration}

3.1. Environment. The analysis presented in this paper is done in a simulated shallow water environment located in the Mediterranean Sea during a summer period. The environment is range independent with a water depth of $130 \mathrm{~m}$. The sound speed profile of the water column (Figure 1) is characterized by a strong negative gradient of approximately $25 \mathrm{~m} / \mathrm{s}$. The highest gradient is located in the first half of the water column. The seabed parameters are presented in Figure 2. Modal functions no. 1 to 9 of the studied environment are presented in Figure 3.

3.2. Signal Sources and Reception Configurations. In this paper we consider two impulsive broadband sources in the ULF band: the first one ULF-1 lasts several tens of milliseconds and has a flat spectrum; the second source ULF-2 lasts several hundreds of milliseconds and is made 


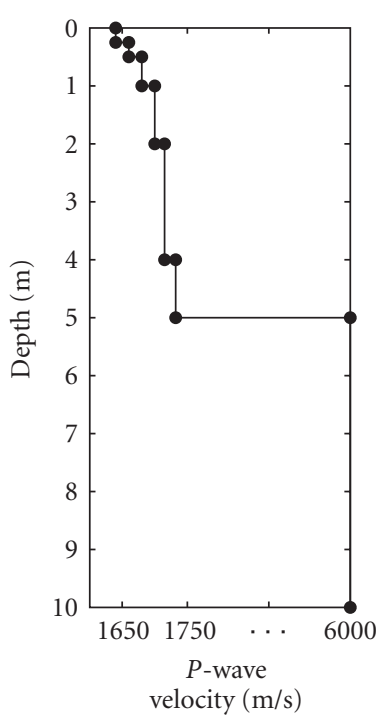

(a)

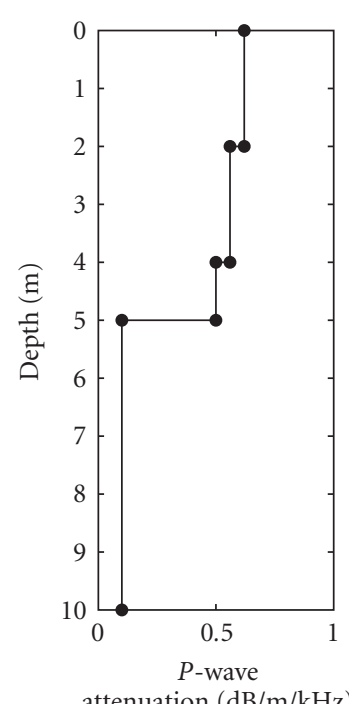

(b)

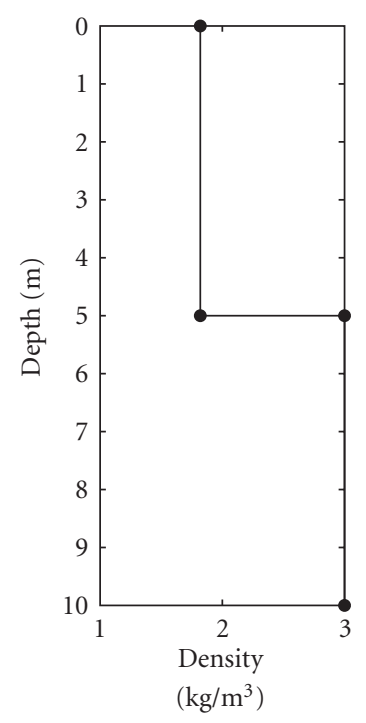

(c)

Figure 2: Vertical structure of the seabed: $P$-wave velocity (a), $P$-wave attenuation (b) and density (c).

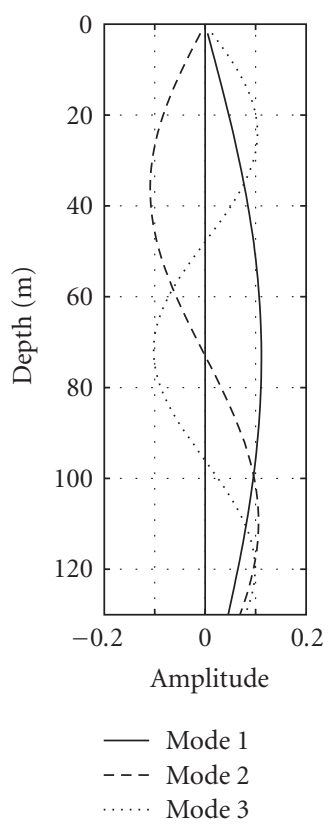

(a)

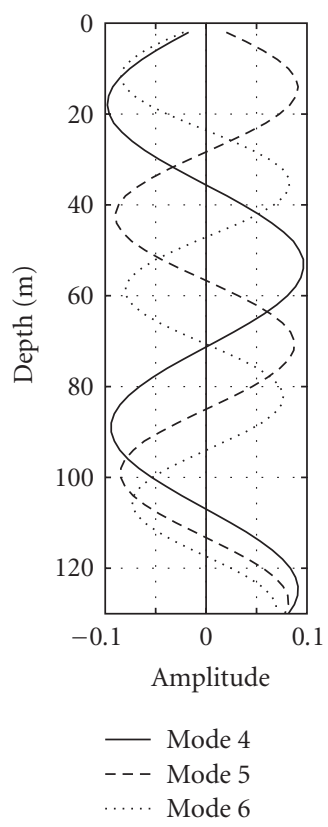

(b)

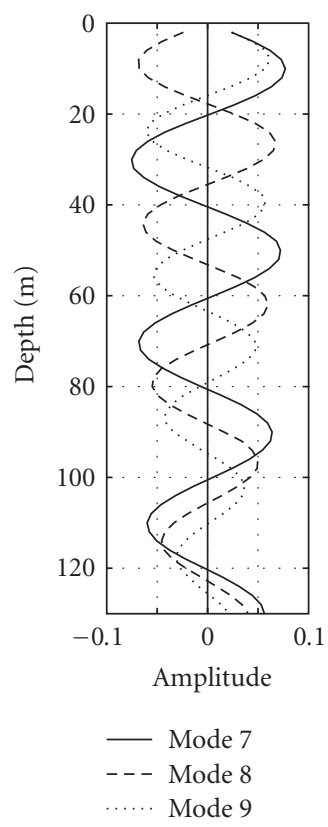

(c)

FIgURE 3: Theoretical modal functions no. 1 to 9 based on Moctesuma's modelization. The frequency-dependent functions are calculated as a mean over frequency band 1 to $100 \mathrm{~Hz}$.

of four band signals (“4 hills" spectrum). Both signals are presented in temporal and spectral domains in Figure 4.

The source ULF-1 is used to validate the methods in a simple case. For a more complex situation source ULF-2 is then used in Section 6.3.

Signals radiated by source are recorded on a horizontal line array (HLA) after acoustic propagation. The HLA is $800 \mathrm{~m}$ long and is composed of 240 omnidirectional equispaced hydrophones (separated by $3.347 \mathrm{~m}$ ). The sampling frequency is $250 \mathrm{~Hz}$.

The experimental configuration is given on Figure 5. Three different source depths $z_{s}$ are studied: $40 \mathrm{~m}, 70 \mathrm{~m}$, and
$105 \mathrm{~m}$. The horizontal distance between the source and the first sensor of HLA is equal to $10 \mathrm{~km}$. The HLA is located on the sea bottom $\left(z_{\text {HLA }}=D\right)$. The HLA can be located at any depth, but this information has to be known. In our simulations the source and the HLA are motionless.

3.3. Data. Moctesuma simulator provides a section of the pressure recorded on the HLA $P\left(r, z_{\mathrm{HLA}}, t\right)$ denoted signal $r-t$ for the sake of simplicity in the following. It is a sampling of the pressure field in radial distance $r$ and in time $t$. The size of the data is defined by 


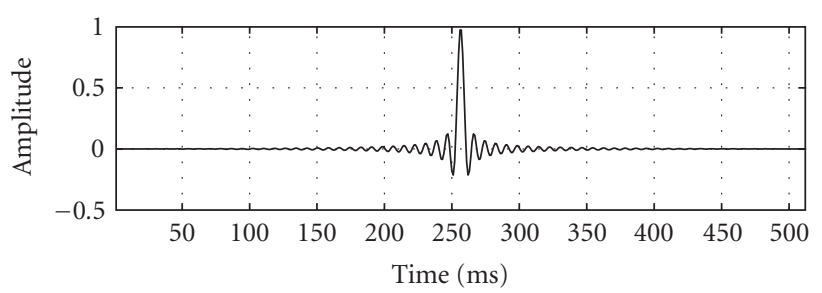

(a)

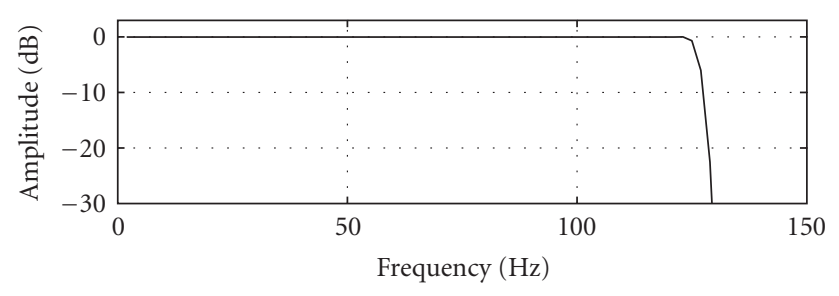

(c)

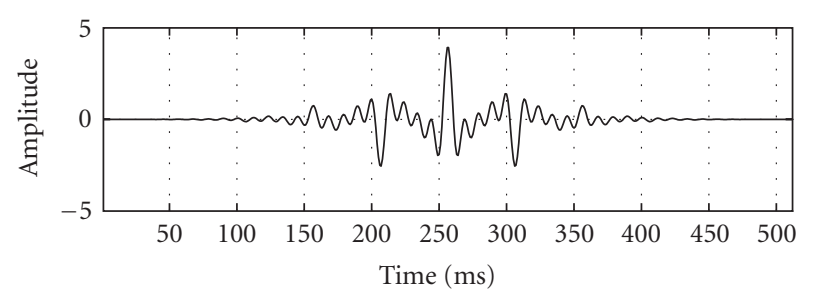

(b)

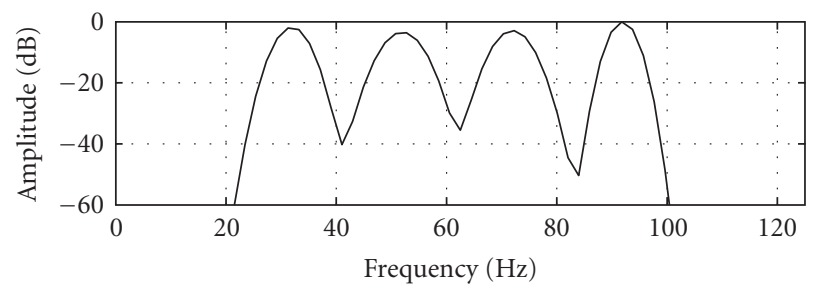

(d)

FIgURE 4: ULF sources: waveforms and spectrums. The first signal (a) and (c), denoted as ULF-1, is a flat spectrum transient. The second signal (b) and (d), denoted as ULF-2, is a broadband comb-type (“4 hills" spectrum) transient.

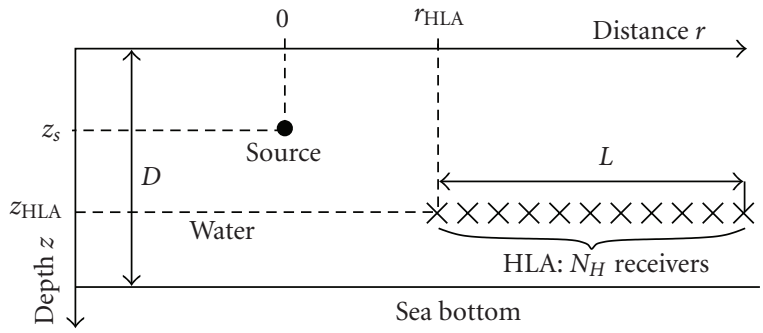

FIgURE 5: Experimental configuration.

(i) number of hydrophones (traces) $N_{r}$;

(ii) duration and frequency sampling of the recording (number of samples $n_{t}$ ).

The "real data" is obtained by adding a white bidimensional (in time and in space) Gaussian noise to the simulated data. Several signal-to-noise ratios (SNRs) are considered.

\section{Filtering Methods}

In this paper, source localization in depth and distance is performed either by multi-dimensional or by monodimensional approach. The first one will be called $F-K$ approach (as the method operates in the frequencywavenumber domain $f-k$ ) and the second one $F T_{a}$ approach (as the method is based on adapted Fourier transform). They both achieve modal filtering which is described in this section.

For the first approach, in the frequency-wavenumber plane $(f-k)$ modes are separated and thus can be filtered. In the second approach, which is theoretically more difficult as we have a single hydrophone, modes are not easily separable and thus, cannot be filtered using classical signal representations such as Fourier transform or time-frequency representation. As proposed in $[12,17]$, we use an adapted frequency representation in which modes are separable and consequently can be filtered.

4.1. Multi-Dimensional Approach. In the multi-dimensional case, the radial distance-time section $P(r, t)$ is represented in the frequency-wavenumber plane $f-k$. The transformation, called $F-K$ transform and denoted by $P\left(k_{r}, f\right)$, is linked to $P(r, t)$ via a two-dimensional Fourier transform (in radial distance $r$ and in time $t$ ). The $F-K$ transform of the signal $P(r, t)$ is defined by

$$
P_{f k}\left(k_{r}, f\right)=\left|\int_{t} \int_{r} P(r, t) e^{-2 i \pi\left(f t-k_{r} r\right)} d t d r\right| .
$$

To improve dynamics of modal representation and avoid spatial aliasing in the $f-k$ plane, a $V_{\text {ref }}$ velocity correction on the section $P(r, t)$ is applied before calculating $F-K$ transform [11] (classical preprocessing technique used in array processing). This operation consists in applying to every trace of the section a time shift, so that the direct wave (traveling with speed $V_{\text {ref }}$, equal to $V_{1}$ in the perfect waveguide) arrives at every sensor at the same time (giving an apparent infinite velocity). Let us denote $X\left(k_{r}, f\right)$ the $F-K$ representation of the section $x(r, t)$. Then, $F-K$ representation of the section after velocity correction $y(r, t)=$ $x\left(r,(t+r) / V_{\text {ref }}\right)$ is

$$
Y\left(k_{r}, f\right)=X\left(\frac{k_{r}+f}{V_{\text {ref }}}, f\right) .
$$

The consequence of this processing is a shifting of every point in the $f-k$ plane in such way that the spatial aliasing is canceled and the representation space of modes is much larger (greater dynamics, simpler filtering). 


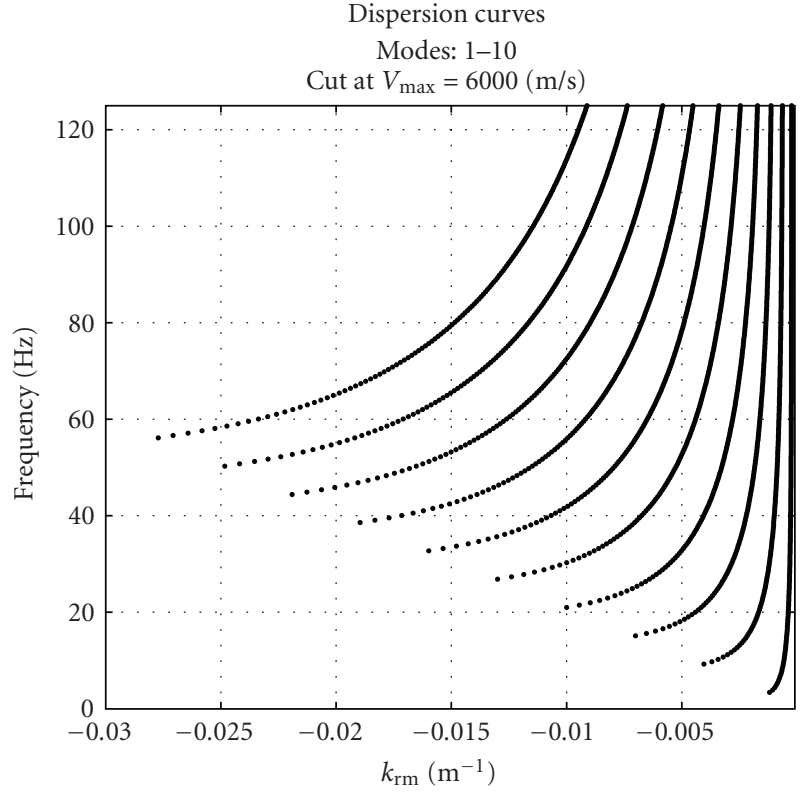

(a)

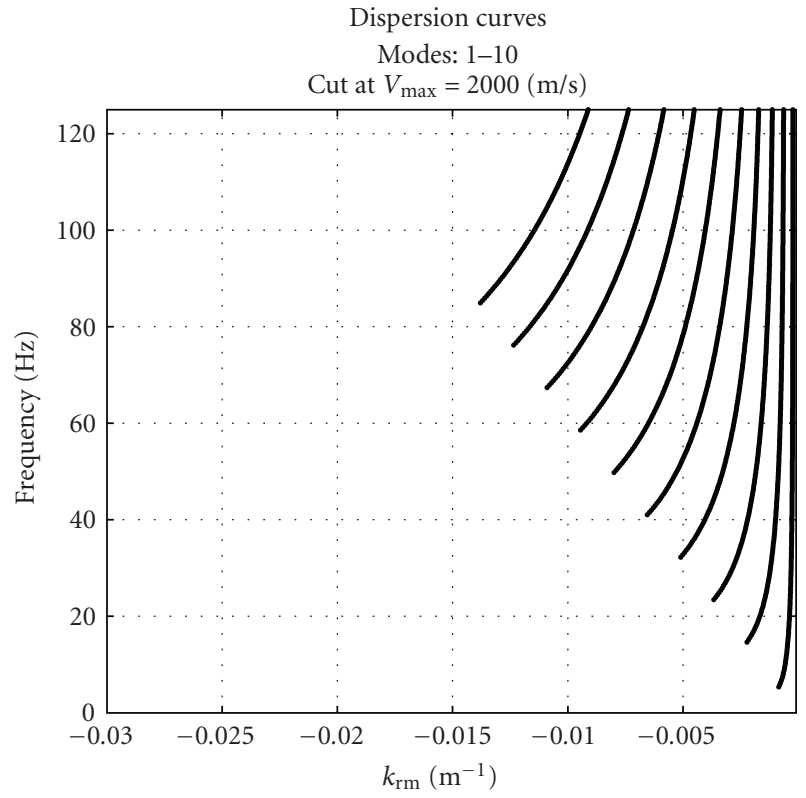

(b)

FIgURE 6: Moctesuma's dispersion curves no. 1 to 10 in frequency-wavenumber domain after $V_{\text {ref }}$ velocity correction (in plot, the mode number increases from (b) to (a)). Wavenumbers in (a) correspond to all propagating modes (water and seabed), and these in (b) plot correspond only to modes propagating in the water column.

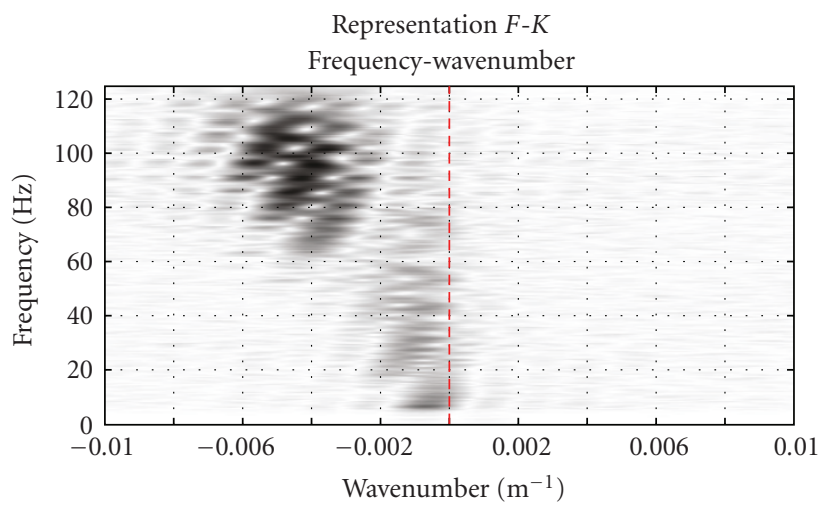

(a)

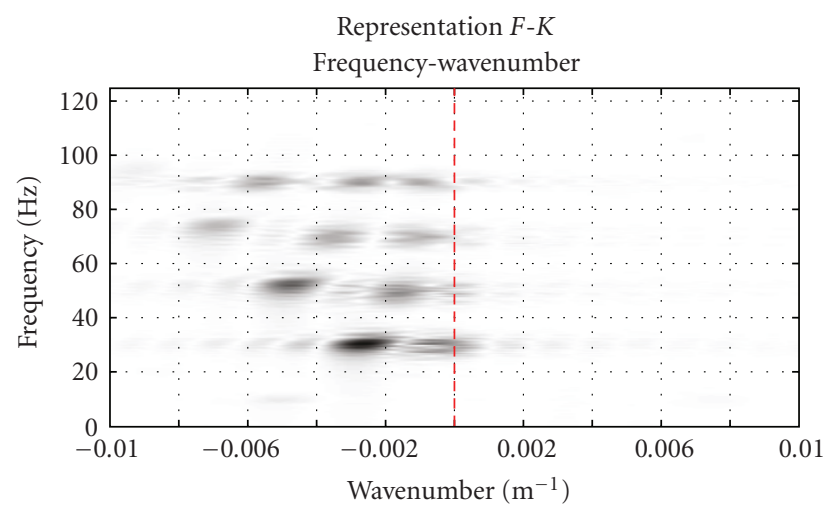

(b)

Figure 7: $F-K$ transforms of sections $r$ - $t$ simulated with source ULF-1 at $105 \mathrm{~m}$ (a) and source ULF-2 at $70 \mathrm{~m}$ (b).

If we consider a white broadband source radiating a transient signal in a perfect waveguide, the $F-K$ transform of the pressure signal (see (2)) received on the HLA, at long range, can be approximated by

$$
P_{f k}\left(k_{r}, f\right) \approx\left|B(f) \cdot \sum_{m=1}^{\infty} \psi_{m}\left(z_{s}\right) \psi_{m}(z) \delta\left(k_{r}-k_{r m}\right)\right|
$$

with $B(f)$ a frequency dependent constant related to the source spectrum.

The theoretical modal signal energy is located on the mode dispersion curves $k_{r}(m, f)=k_{r m}(f)$ (the form of the mode dispersion curves is given in Figure 6). As one can notice, for each frequency the wavenumber spectrum is discrete. The $F-K$ representations of two data sets are given in
Figure 7. The first plot shows a simulation done with signal ULF-1 (flat spectrum) at depth $105 \mathrm{~m}$. The energy is spread across all the frequency band. The second plot presents a simulation with signal ULF-2 ("4 hills" spectrum) at depth $70 \mathrm{~m}$. The $F-K$ representation reflects exactly the spectrum of the source signal (see Figure 4).

As the HLA is located at a known depth, values of the factors $\psi_{m}(z)$ are known. For HLA located on the sea bottom $\left|\psi_{m}(z)\right| \approx 1$ and expression (7) can be rewritten as

$$
P_{f k}\left(k_{r}, f\right) \approx|B(f)| \cdot \sum_{m=1}^{\infty}\left|\psi_{m}\left(z_{s}\right)\right| \cdot \delta\left(k_{r}-k_{r m}\right) .
$$

The amplitude of the $F-K$ transform for each curve (dispersive mode) depends only on the mode excitation factor 
modulus. We use these curves to estimate the excitation factor modulus of each mode. For a perfect waveguide model there is no frequency dependence for modal functions, which is the case in reality. Therefore, excitation factor of mode $m$ is estimated as the mean value across the frequency domain. Moreover, mode excitation factors at the bottom interface are not exactly equal to 1 and will slightly modify the estimation of the mode amplitude at the source depth. This phenomenon does not affect the result as the same methodology is applied for the replica data.

Mask Construction. Once $F-K$ representation of the signal is calculated, a mask filtering has to be applied to filter modes. The mask is a binary image (with the same size as the $F-K$ transform) and is used to extract a mode by a simple multiplication in the $f-k$ domain. The mask built for each mode should "cover" the region occupied by this mode in the $f-k$ plane.

An initial mask of mode $m$ is created using its wavenumbers. These can be computed theoretically by a propagation model if the environmental parameters are known. In our case they are given by Moctesuma (see Figure 6). Then, the mask of mode $m$ is dilated independently in both domains (frequency and wavenumber) with the dilation parameter $d_{k, f}^{m}=\left[d_{k}^{m}, d_{f}\right]$ according to the following formula:

$$
M_{m}\left(k_{r}, f\right)=\sum_{d_{k}^{m}} \sum_{d_{f}} \delta\left(k_{r}-k_{r m}\left(f \pm d_{f} \cdot \Delta f\right) \pm d_{k}^{m} \cdot \Delta k\right),
$$

where $d_{k}^{m}$ and $d_{f}$ denote, respectively, dilation sizes in wavenumber $\left(d_{k}^{m} \in\left\{1, \ldots, d_{k_{\max }}^{m}\right\}\right)$ and frequency $\left(d_{f} \in\right.$ $\left.\left\{1, \ldots, d_{f_{\max }}\right\}\right)$ domains, and $\Delta k$ and $\Delta f$ denote, respectively, the sampling period in wavenumber and frequency domains. The first parameter $d_{k}^{m}$ determines the distances between successive masks (depends on mode number $m$ ) and the second parameter $d_{f}$ defines the distance of the mask for mode 1 to the frequency $0 \mathrm{~Hz}$. This definition of dilation parameters makes the mask width in the frequency dimension adapted to the frequency (narrower masks at high frequencies for lower number modes and larger masks at lower frequencies for higher number modes). The dilation process is restricted by limitation that the masks for different modes must not overlap. These masks allow an efficient filtering even for higher modes which are usually more difficult to filter.

The simulated environment has to be as close as possible to the real environment to achieve a good filtering of modes. Moreover, it is necessary to dilate the previous theoretical mask for two reasons:

(i) the limited HLA length-mode energy spreads around dispersion curves in the $f-k$ plane;

(ii) the mismatch between real and simulated environments [11].

A total set of masks for the studied environment is given in Figure 8. This set contains 14 masks which corresponds to the total number of propagating modes in this environment. An example of the mask adapted to mode no. 4 is given in

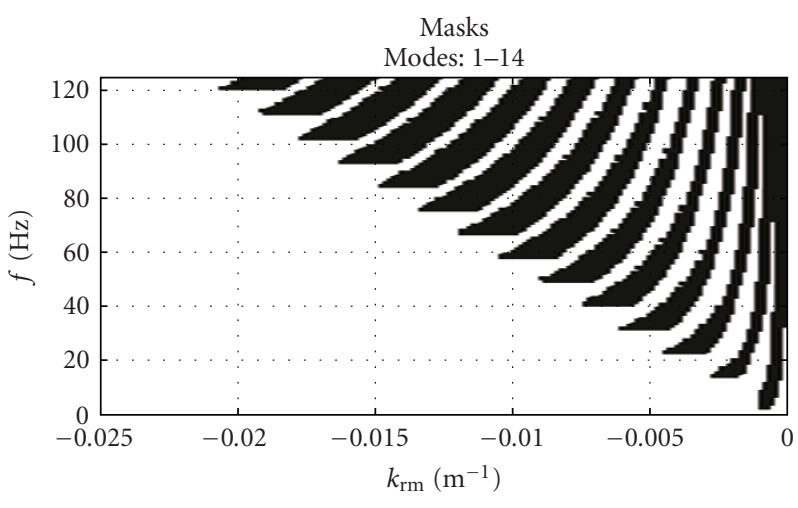

FIGURE 8: Set of binary masks in the $f-k$ domain built from dispersion curves given in Figure 6 by bi-dimensional dilatation process. The set is complete for the studied environment.

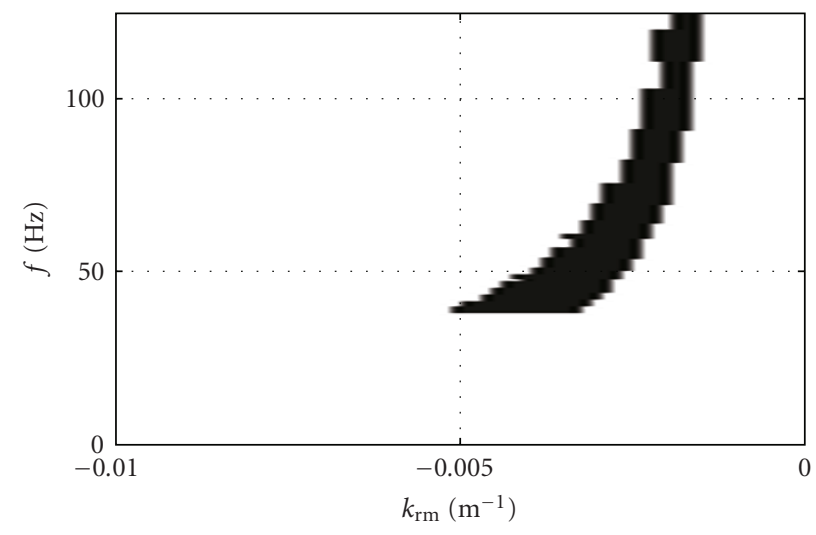

Figure 9: Binary mask used to filter mode no. 4.

Figure 9. The mask of mode no. 4 is built starting from its dispersion curve (see Figure 6, 4th trace counting from right to left) and then dilated according to (9).

The energy spectrum of data in Figure 7 (a) is shown in Figure 10. For each mode the energy is calculated as a mean of the $f-k$ region where this mode is present (after mask filtering).

In Figure 11 we present the result of the mode filtering for modes nos. 1, 4 (mode with very low energy), and 6 (the mode with the highest energy).

4.2. Monodimensional Approach. In the mono-dimensional configuration, classical signal representations such as Fourier transform or short-time Fourier transform are not suited for description of a signal that can be decomposed in a sum of dispersive modes (nonstationary and nonlinear timefrequency patterns). Therefore, these techniques cannot correctly represent the signal having modal structures (see Figures 12(a) and 13(a)). This signal processing problem has attracted much interest for the last decades $[13,18,19]$. In this paper we based our monosensor approach on works $[12,17,20]$. The idea is to find a representation well adapted for modal signal structure to achieve modal filtering. The best way is to take into account the physics of oceanic 


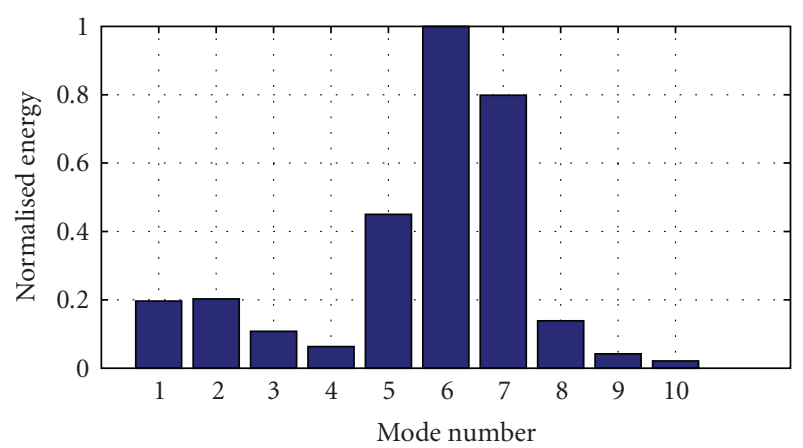

FIGURE 10: Energy spectrum in modal space (for modes no. 1 to 10).

waveguide propagation and build a representation adapted to the signal structure in the same way as Fourier transform is adapted to a monochromatic signal. Here, we discuss only an adapted frequency representation $F T_{a}$. However, on the same rule an adapted time-frequency representation can be constructed and used $[12,17]$.

The adapted processing tools are based on the combination between acoustic wave propagation (waveguide propagation law) and signal processing theory (unitary equivalence). Building of the adapted frequency representation is based primary on definition of the unitary operator of transformation adapted to guided waves. This unitary operator is linked to the dispersion law $v_{m}=f(t)$, where $v$ is the instantaneous frequency and $t$ is the time. For a perfect waveguide model and for each mode $m$ the dispersion law is defined by

$$
\nu_{m}^{\mathrm{parf}}(t)=\frac{(2 m-1) V_{1} t}{\sqrt{4 D\left[t^{2}-\left(r / V_{1}\right)^{2}\right]}},
$$

where $V_{1}$ is the sound speed in water, $D$ is the waveguide depth, $t$ is the time, and $r$ is the distance. This relation defines temporal domain of group delay $\mathscr{D}_{f}=\left(R^{+} / V_{1},+\infty\right]$, where $R^{+} / V_{1}$ is arrival time of the wavefront.

Starting from the dispersive relation given in 10 , the instantaneous frequency $v_{m}$ is the derivative of the instantaneous phase $\phi_{m}^{\text {parf }}(t)$ :

$$
\begin{aligned}
\phi_{m}^{\mathrm{parf}}(t) & =2 \pi \int_{t} \nu_{m}^{\mathrm{parf}}(t) d t \\
& =2 \pi \frac{(2 m-1) V_{1}}{4 D} \sqrt{\left[t^{2}-\left(\frac{r}{V_{1}}\right)^{2}\right]} \\
& =2 \pi v_{c}(m) \xi(t)
\end{aligned}
$$

with $v_{c}(m)$ the cut-off frequency of mode $m$ defined by

$$
\nu_{c}(m)=\frac{(2 m-1) V_{\mathrm{ref}}}{4 D} .
$$

$\xi(t)$ is called the general dispersive function and is defined as follows:

$$
\xi(t)=\sqrt{\left[t^{2}-\left(\frac{r}{V_{1}}\right)^{2}\right]}
$$

As a result, the pressure signal of the perfect waveguide model is defined by

$$
p^{\mathrm{parf}}(t)=\sum_{m} g_{m}(t) e^{2 \pi i v_{c}(m) \xi(t)}
$$

with $g_{m}(t)$ the envelope evolution of mode $m$. The unitary operator of transformation should transform nonlinear mode structures in linear ones (in time-frequency domain), and thus allow the use of classical Fourier filtering techniques. In construction of such representation, the unitary equivalence formalism is used [13]. One of the unitary operators is a warping operator $\mathbf{W}_{w}$ (applied to a signal $x(t)$ ) defined as follows:

$$
\left(\mathbf{W}_{w} x\right)(t)=\left|\frac{\partial w(t)}{\partial t}\right|^{1 / 2} \cdot x[w(t)] .
$$

The function $w(t)$ has to be derivable and bi-univocal, and function $w^{-1}(t)$ has to exist. The operator $\mathbf{W}_{w}$ is applied to the signal in order that $\left(\mathbf{W}_{w} p^{\text {parf }}\right)(t)$ becomes a sum of linear structures

$$
\left(\mathbf{W}_{w} p^{\text {parf }}\right)(t)=\sum_{m}\left|\frac{\partial w(t)}{\partial t}\right|^{1 / 2} C_{m} e^{2 \pi i v_{c}(m) \xi[w(t)]} .
$$

To do so, we deduce from (16), the deformation function $w(t)$ defined on $\mathbf{R}^{+} \rightarrow \mathscr{D}_{f}$

$$
w(t)=\xi^{-1}(t)=\sqrt{\left(t^{2}+\frac{r^{2}}{V_{1}^{2}}\right)} .
$$

Finally, the unitary operator of transformation adapted to a perfect waveguide is

$$
\left(\mathbf{W}_{w} p^{\text {parf }}\right)(t)=\sum_{m}\left|\frac{\partial w(t)}{\partial t}\right|^{1 / 2} C_{m} e^{2 \pi i v_{c}(m) t} .
$$

Note that this tool is reversible, so one can go back to the initial representation space (time or frequency).

In this short presentation of adapted transformation for the perfect waveguide we demonstrated the principal idea of this technique which consists in transformation of modes into linear structures. In our work we use a method adapted to Pekeris waveguide model, as it is a more complex model (closer to reality) taking into account the interaction with the sea bottom (described in details in [12]).

As the non-linear time-frequency signal structures become linear after this transformation, the signal becomes stationary (see Figures 12(b) and 13(b)). In this case, one can use classical frequency filtering tools to filter modes. The modal filtering is then done in the adapted frequency domain (Pekeris frequency) by a simple bandpass filtering defined by the user.

\section{Estimators}

In Section 4 we demonstrated how extracting modes from the recorded signal by multi and mono-dimensional approaches. In this section we discuss depth and distance 


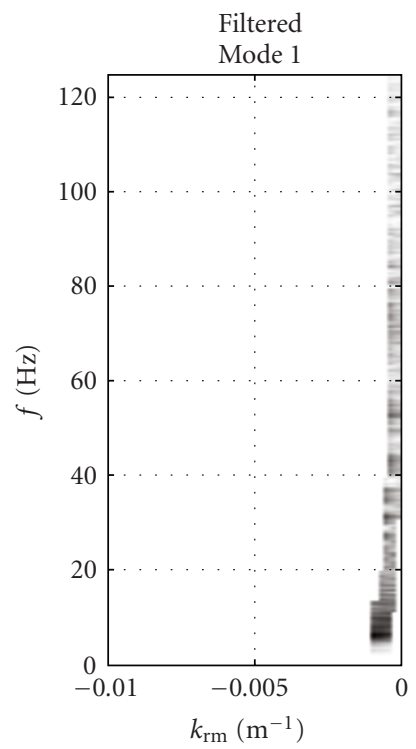

(a)

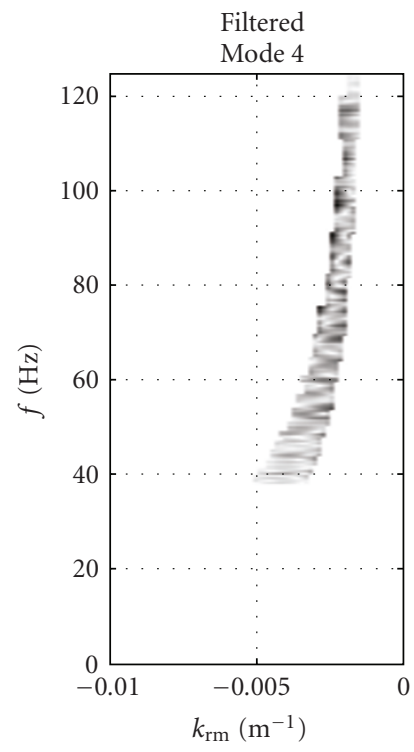

(b)

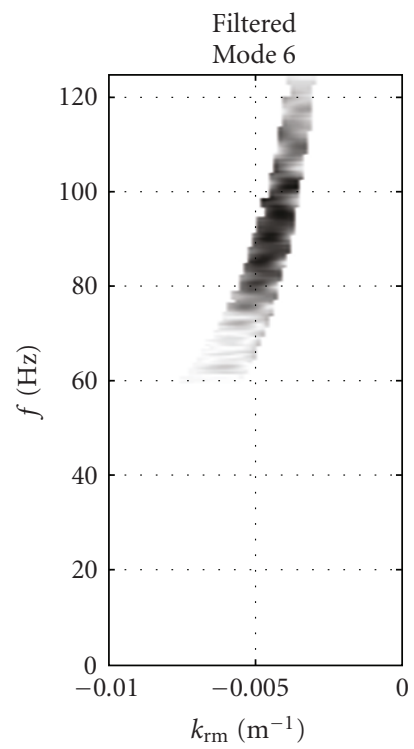

(c)

Figure 11: Modes nos. 1, 4, and 6 extracted from $F-K$ representation given in Figure 7(a) by mask filtering.

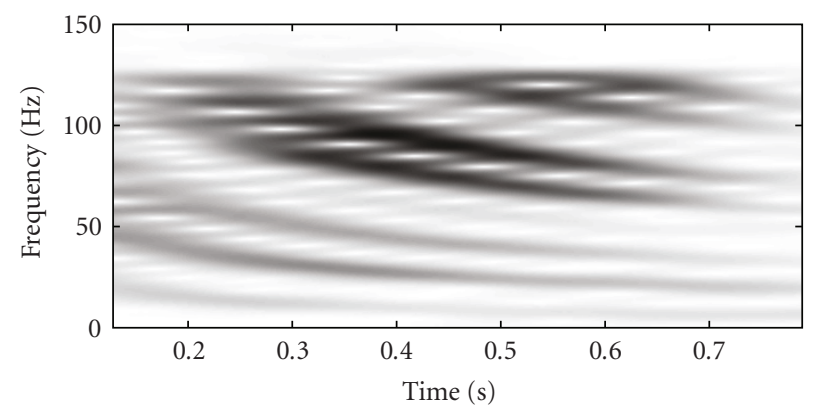

(a)

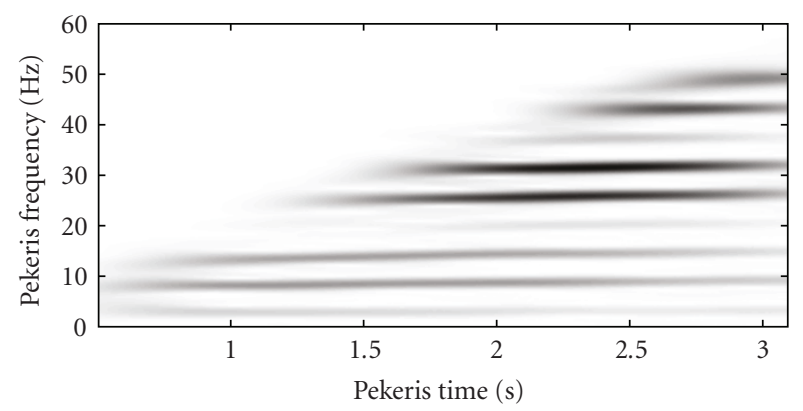

(b)

FIGURE 12: Classical STFT and adapted STFT (matched to Pekeris model) of a signal recorded on a single hydrophone. The simulation is given for the source ULF-1 at 40-m depth and $10 \mathrm{~km}$ distant from hydrophone.

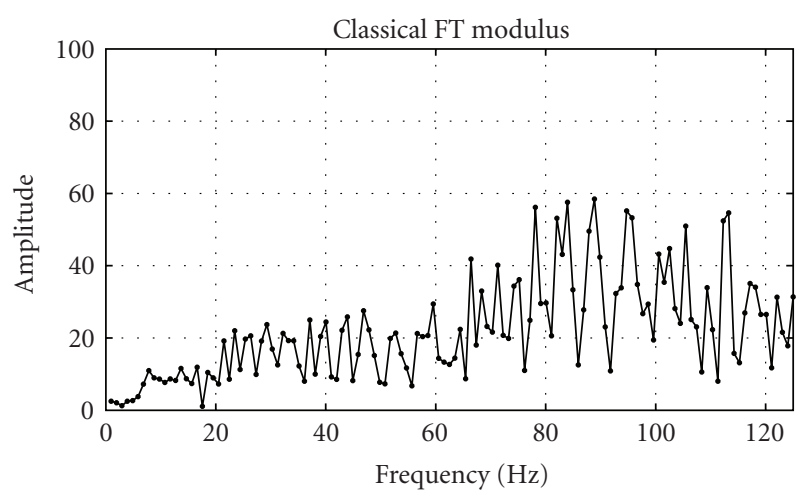

(a)

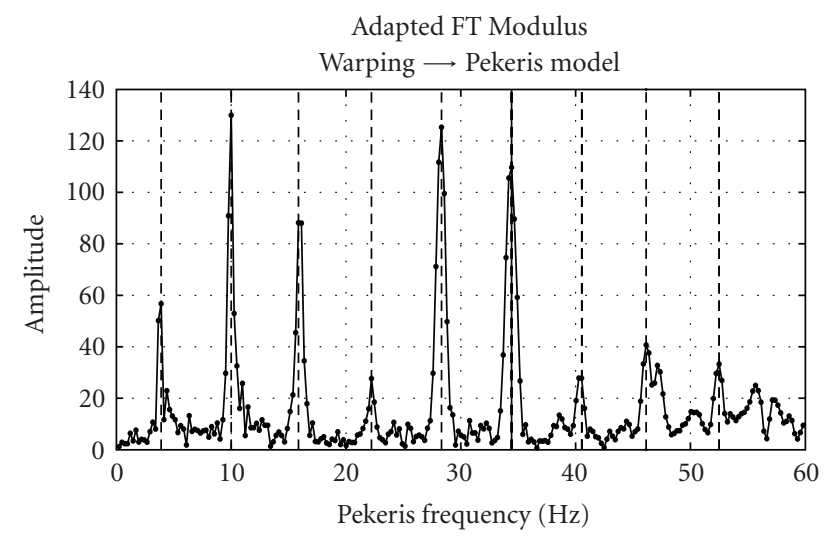

(b)

FIGURE 13: Classical Fourier spectrum and adapted Fourier spectrum of a signal recorded on a single hydrophone (the same as in Figure 12). 
TABLE 1: Values of $\Delta \phi(m, n, z)$.

\begin{tabular}{lccc}
\hline & & \multicolumn{2}{c}{ Sign of $\psi_{n}(z)$} \\
\hline \multirow{3}{*}{ Sign of $\psi_{m}(z)$} & & + & - \\
& + & 0 & $\pi$ \\
& - & $\pi$ & 0 \\
\hline
\end{tabular}

estimators based on modal processing. For depth estimation, we use a matched-mode technique, and for distance estimation our approach is based on mode phase analysis. For both approaches, $F-K$ and $F T_{a}$, we use the same estimators for localization in depth and distance. The only difference is the representation space of modal filtering: frequencywavenumber for $F-K$ method and adapted Fourier spectrum for $F T_{a}$ method.

5.1. Range Estimation. The range estimation is combined with the mode sign estimation; therefore we call the estimator sign-distance estimator. The estimator applied only on the real data is based on mode phase analysis and calculates a cost function $C$ based on two mode phases ( $m$ and $n$, where $n>m$ ) extracted from the data. Modes are not necessarily consecutive; however their numbers and associated wavenumbers (calculated by Moctesuma) have to be known.

The sign-distance estimator is originally based on a work published in [20]. We present this estimator very succinctly, more details can be found in [21]. The principle is based on the definition of mode phase given in (4). To suppress the unknown parameters in this equation, the initial estimator $\Delta \Phi$ uses the difference between two mode phases:

$$
\begin{aligned}
\Delta \Phi(m, n, f) & =\Phi_{m}(f)-\Phi_{n}(f) \\
& =\Delta \phi\left(m, n, z_{s}\right)+\Delta \phi\left(m, n, z_{\mathrm{HLA}}\right)+r \Delta k_{r}(m, n, f)
\end{aligned}
$$

with

(i) $\Delta \phi(m, n, z)$ being difference between two mode phases at depth $z$; the values of this parameter are defined by (for details see Table 1):

$$
\begin{aligned}
\Delta \phi(m, n, z) & =\phi\left(\psi_{m}(z)\right)-\phi\left(\psi_{n}(z)\right) \\
& =\pi \delta_{\operatorname{sign}\left(\psi_{m}(z)\right),-\operatorname{sign}\left(\psi_{n}(z)\right)}(\bmod (2 \pi)),
\end{aligned}
$$

(ii) $\Delta k_{r}(m, n, f)=k_{r m}(f)-k_{r n}(f)$.

The estimation is done in sequential way; that is, superior mode signs ( of order $n$ ) are estimated using inferior mode signs (of order $m$ ). The starting point is the absolute mode sign of mode no. 1 which is always positive: $\widehat{S}_{\mathrm{abs}}\left(1, z_{s}\right) \rightarrow+$. For example to estimate the sign of mode $n=5$, one can use in theory modes $m=\{1,2,3,4\}$. As the frequency band shared by two modes ( $m$ and $n$ ) has to be maximized for the estimation performance, we propose the following rule to
TABLE 2: Absolute mode sign choice rule: the absolute sign of mode $n \hat{S}_{\text {abs }}\left(n, z_{s}\right)$ depends on the absolute sign of mode $m \hat{S}_{\text {abs }}\left(m, z_{s}\right)$ and the pass function $\widehat{\Delta \phi}\left(m, n, z_{s}\right)$ (where $\left.n>m\right)$.

\begin{tabular}{lcc}
\hline$\hat{S}_{\mathrm{abs}}\left(m, z_{s}\right)$ & $\widehat{\Delta \phi}\left(m, n, z_{s}\right)$ & $\hat{S}_{\mathrm{abs}}\left(n, z_{s}\right)$ \\
\hline+ & 0 & + \\
+ & $\pi$ & - \\
- & 0 & - \\
- & $\pi$ & + \\
\hline
\end{tabular}

choose inferior mode number $(m)$ for estimation of superior sign mode number $(n)$ :

$$
m=\{n-3, n-2, n-1\} .
$$

The sign of $\Delta \phi\left(m, n, z_{\mathrm{HLA}}\right)$ is known as the depth $z$ of HLA is known (in our case it is $D$ ). To estimate mode signs for real data, for each frequency $f$ the quantity $\Delta \Phi_{\exp }$ is measured, and then we calculate the cost function $C$ defined as

$$
C\left(r, \Delta \phi\left(m, n, z_{s}\right)\right)=\sum_{f} \mid d\left(\Delta \Phi_{\exp }(m, n, f),\left.\Delta \Phi(m, n, f)\right|^{2},\right.
$$

where $d$ is the distance function defined as

$$
d\left(\phi_{1}, \phi_{2}\right)=\arg \left(\exp \left(i\left(\phi_{1}-\phi_{2}\right)\right)\right)
$$

with arg defined on a basic interval $(-\pi, \pi]$. The signdistance estimator is found by the minimization of the cost function $C$ :

$$
\left\{\widehat{r}, \widehat{\Delta \phi}\left(m, n, z_{s}\right)\right\}=\underset{r, \Delta \phi\left(m, n, z_{s}\right)=\{0, \pi\}}{\arg \min } C\left(r, \Delta \phi\left(m, n, z_{s}\right)\right),
$$

where $r \in\left\langle r_{\min }, r_{\max }\right\rangle$ and

$\widehat{\Delta \phi}\left(m, n, z_{s}\right)= \begin{cases}0 & \text { if modes } m \text { and } n \text { have the same signs, } \\ \pi & \text { if modes } m \text { and } n \text { have opposite signs. }\end{cases}$

The sign-distance estimator is calculated for 2 possible values of $\widehat{\Delta \phi}\left(m, n, z_{s}\right): 0$ and $\pi$. By minimizing $C$, we find the searched value of $\widehat{\Delta \phi}\left(m, n, z_{s}\right)$ (using (25), the relative sign between modes $m$ and $n$ is estimated) and also the distance. As we know the absolute sign $\hat{S}_{\mathrm{abs}}\left(m, z_{s}\right)$ of inferior mode $m$ (known or estimated in previous step of estimation) and the estimated value of the pass function $\widehat{\Delta \phi}\left(m, n, z_{s}\right)$ betwen modes $m$ and $n$, we can find the absolute sign of mode $n \rightarrow \widehat{S}_{\mathrm{abs}}\left(n, z_{s}\right)$. The rule of mode sign estimation is given in Table 2.

The mode sign estimation for $N_{\bmod }=K$ takes at least $K-1$ steps as the first mode sign is always positive and as the estimator works sequentially on mode couples.

5.2. Depth Estimation. Source depth estimation is based on Matched-Mode Processing. The principle of MMP is 


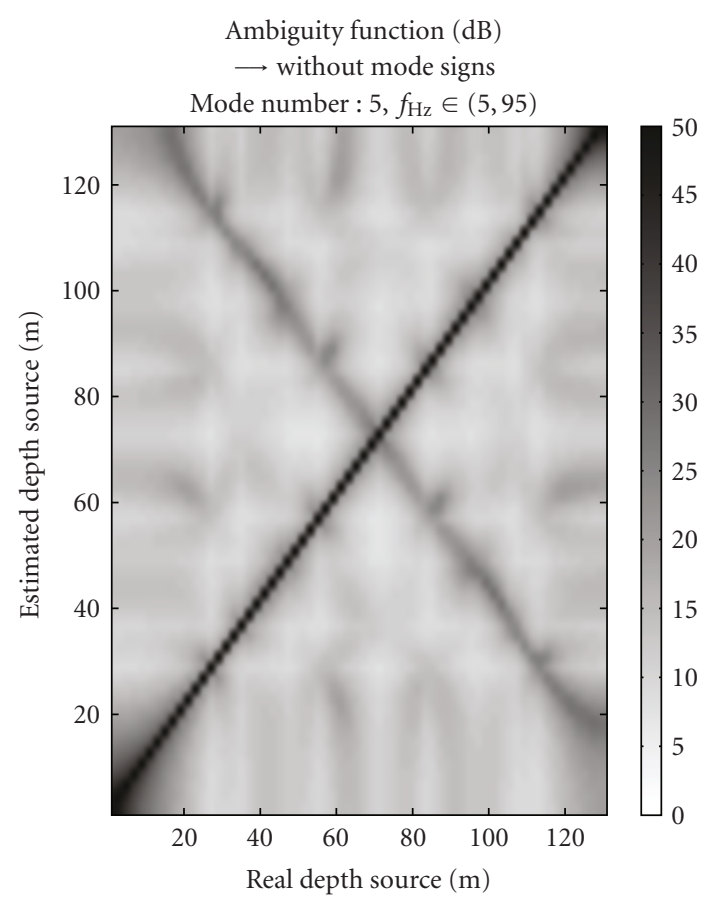

(a)

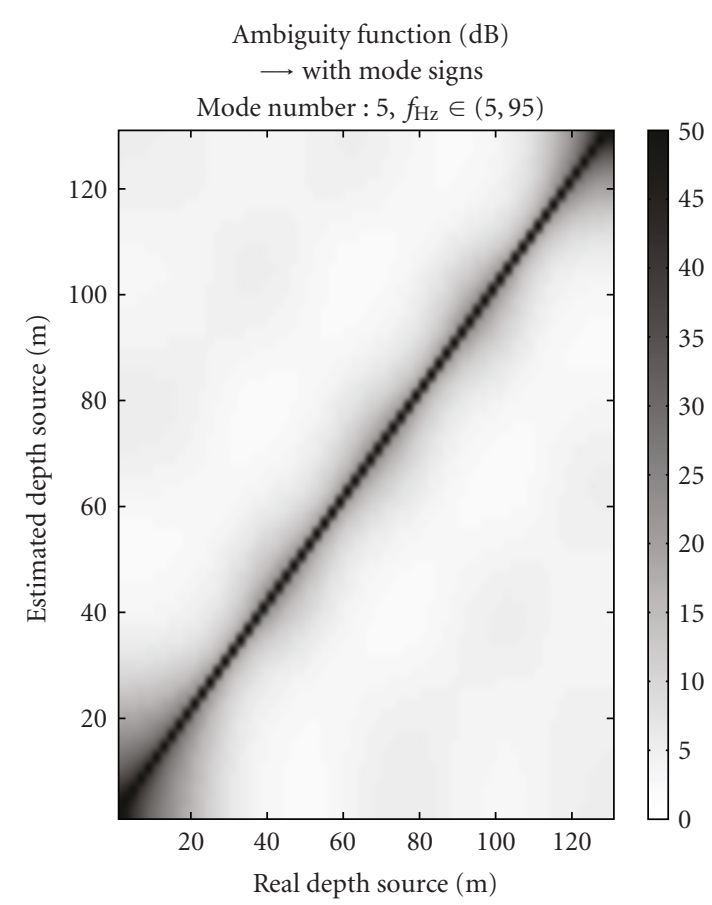

(b)

FIgURE 14: Theoretical ambiguity functions for matched-mode localization. Results obtained for the set of modes 1 to 5 and frequency band $(5,95) \mathrm{Hz}$. In (a) the contrast function $G$ is calculated at the basis of mode excitation factor modulus, and in (b) the mode sign information is integrated.

to compare modes in terms of excitation factors extracted from the real data with those extracted from the replica fields. The modeled acoustic field (replica) is simulated with Moctesuma. The depth estimator is based on a correlation which measures a distance between mode excitation factors estimated from real and from simulated data (for a set of investigated depths). The depth for which this correlation reaches maximum (the best matching) is chosen as the estimated source depth.

The mode excitation factors are extracted by $F-K$ or $T F_{a}$ approach on real and replica data in the same manner. They are positive as they are extracted from positive value spaces $(F-K$ transform or modulus of the adapted Fourier transform). Their signs are then obtained using the signdistance estimator presented in the previous section. The mode signs are estimated only for real data. For the reference data, as the simulations are done for a set of determined source depths, mode signs are known. The combination of mode excitation factors with mode signs allows canceling secondary peaks in the correlation function. These peaks are due to "mirror solutions" of modal functions for some depths when considering only mode excitation factor modulus (see Figure 14). Therefore, adding mode sign information to mode excitation factor modulus improves significantly the performance of depth localization (shown on examples in Section 6).

The localization performance is strongly dependent of the matching accuracy between real and simulated acoustic fields. Study of the influence of environmental and system effects on the localization performance is presented in [11, 22]. The dilation used to build masks in the $f$ - $k$ plane makes the method more robust against these errors.

To compare mode excitation factors extracted from real and simulated data, a normalization based on the closure relationship is applied:

$$
\sum_{m} c_{m}^{2}\left(z_{s}\right)=1
$$

where $c_{m}$ is an excitation factor of mode $m$. Then, the comparison between mode excitation factors extracted from the real data $c_{m}^{\text {real }}$ and replica data $c_{m}^{\text {simul }}$ is made using the contrast function $G$ :

$$
G_{N_{\text {mod }}}\left(z_{s}\right)=-10 \log \left(\frac{\sum_{m=1}^{N_{\text {mod }}}\left(c_{m}^{\text {real }}-c_{m}^{\text {simul }}\left(z_{s}\right)\right)^{2}}{N_{\text {mod }}}\right),
$$

where $N_{\text {mod }}$ is the number of analyzed modes

$$
\hat{z}_{s}=\underset{z_{s}}{\arg \max } G_{N_{\bmod }}\left(z_{s}\right) .
$$

The maximum of $G$ indicates the estimated depth of the source.

In matched-mode localization, modes for which the function $G$ defined by (27) is calculated are theoretically unrestricted. However, in case of ULF localization, only the first modes are used. The upper mode number limit is given by the environment and existence of cut-off frequencies as 
the methods presented in this paper are based on broadband signal processing. In our analysis the number of used modes is between 5 and 7 .

Theoretical performances of depth localization for the studied environment and for all source depths are presented in Figure 14 (each vertical line corresponds to a contrast function $G$ ). The figure presents two plots: for the method without mode signs (a) and for the method with mode signs (b). The result is obtained by the application of (27) to mode excitation factors directly taken from Moctesuma simulations. For the method without mode signs, one can notice the existence of "mirror solutions" which is a line of secondary peaks intersecting with the primary peaks line indicating the true source positions. That line does not exist for the method with mode signs, as the "mirror solutions" are cancelled by adding mode signs to mode excitation factor modulus. In such way, one can remove the localization ambiguity, which is problematic especially in low signal-tonoise conditions.

5.3. Source Spectrum Estimation. To perform matched-mode localization, knowledge (at least partial) of quantities such as geoacoustical parameters of the environment and spectral characteristics of the source is crucial. This results from the fact that the simulated acoustic field should be simulated in geoacoustical conditions similar (as much as possible) to the real conditions existing in the location of interest. In general, environmental parameters can be estimated using inversion methods $[23,24]$. Unluckily, in passive approach the knowledge of the source spectrum remains notwithstanding problematic. As the influence of the source spectrum is relevant (see (2) and (7)), we propose an estimator of source spectrum based on the analysis of the first mode (most horizontal), as this mode is always excited. The property of distinct attenuation of signal frequencies (growing non linearly with the frequency) is taken into account by the estimator in order to improve estimation quality. The proposed estimator can be formulated as follows:

$$
\hat{X}_{s}(f)=A(f) \cdot \frac{1}{N_{r}} \sum_{n=1}^{N_{r}} X_{n}^{\bmod \{1\}}(f),
$$

where $\hat{X}_{s}(f)$ is the estimated source spectrum, $A(f)$ is a spectral factor correcting the signal attenuation over frequency, $N_{r}$ is a number of hydrophones, and $X_{n}^{\bmod \{1\}}(f)$ is the spectrum of the first mode on hydrophone $n$ estimated from the $f-k$ plane. For better performance the correction factor $A(f)$ can be measured in the field (by recording a known broadband signal at some distance). As we do not operate on real field data, to calculate $A(f)$ we use theoretical values of spectral attenuation (for frequency range of interest).

\section{Localization}

We present some examples of source localization using methods described in Sections 4 and 5. First, examples of localization in distance and in depth are presented using a single hydrophone, and then using a horizontal hydrophone array (HLA). Moreover, we show the interest of mode signs and source spectrum estimations in case of depth localization by $F-K$ and $F T_{a}$ approaches. Due to limited paper's length, we do not expose here the study of the robustness of the methods against noise. These considerations have been studied in $[11,25]$. We give only some most important conclusions. The simulations on source depth estimation demonstrate that to obtain the primary peak-to-secondary peak ratio of $10 \mathrm{~dB}$ the signal-to-noise ratio has to be superior to $-5 \mathrm{~dB}$ for $F-K$ method and $5 \mathrm{~dB}$ for $F T_{a}$ method. The impact of noise on source range estimation seems to be more relevant. These considerations concern white (in time and in space) gaussian model of local (non propagating) noise.

6.1. One Hydrophone. The objective of this section is to show performance of $F T_{a}$ localization method using a single hydrophone. The methods are validated for the environment and configuration described in Section 3 for a signal-to-noise ratio of $15 \mathrm{~dB}$. The distance between source and hydrophone is equal to $10 \mathrm{~km}$. Source is deployed at $40 \mathrm{~m}$ of depth and the hydrophone is on the seabed.

We first apply the deformation of the signal described in Section 4.2 for the Pekeris model. The parameters used to warp the modal signal are

(i) water column depth: $130 \mathrm{~m}$;

(ii) sound speed velocity in water: $1500 \mathrm{~m} / \mathrm{s}$;

(iii) sound speed velocity in sediments: $2000 \mathrm{~m} / \mathrm{s}$;

(iv) water density: $1 \mathrm{~kg} / \mathrm{m}^{3}$;

(v) sediment density: $2 \mathrm{~kg} / \mathrm{m}^{3}$.

Within the parameters, the water column depth is a correct value, and other parameters are approximations of the real values to demonstrate robustness of the method.

Then, the $F T_{a}$ method allows a filtering of modes (classic bandpass filter applied on spectral representation given in Figure 13(b)), and these modes are analyzed for distance and depth estimation.

6.1.1. Distance. For the distance localization an access to mode phases is essential. First, a modal filtering by $F T_{a}$ is performed and then for each analyzed mode, its phase is calculated through a Fourier transform. Wavenumbers needed by the estimator defined in (19) are provided by Moctesuma.

In Figure 15 we show the cost function $C$ corresponding to several distance estimations. As the distance estimator works on mode couples, we present five distance estimations for mode couples: $(2,1),(3,1),(4,2),(5,3)$, and $(6,4)$ and research area $r \in(8,13) \mathrm{km}$ with a step $\Delta r=25 \mathrm{~m}$. The estimated distances are given in Table 3 (the real distance is $10 \mathrm{~km})$. The first 4 estimations are correct, and the last one is false, which is due to limited frequency band of mode 6 . Moreover, the sign-distance estimator provides mode signs. For this example, the mode signs were estimated on the same mode couples as distance. The estimation of mode signs no. 


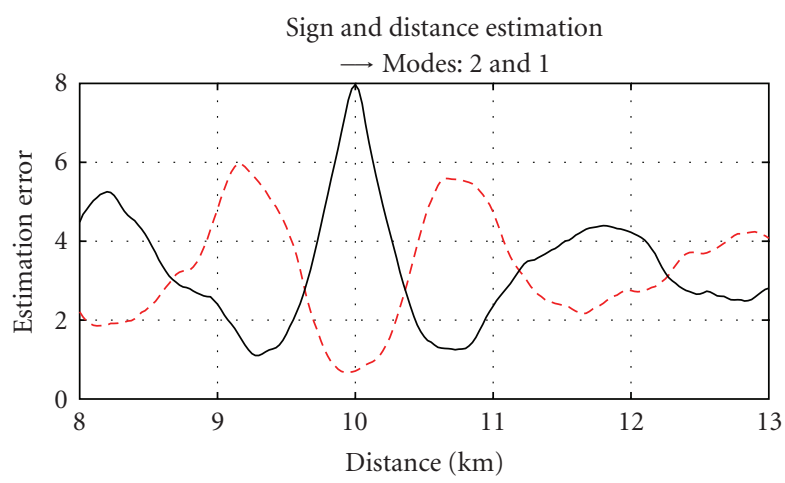

(a)

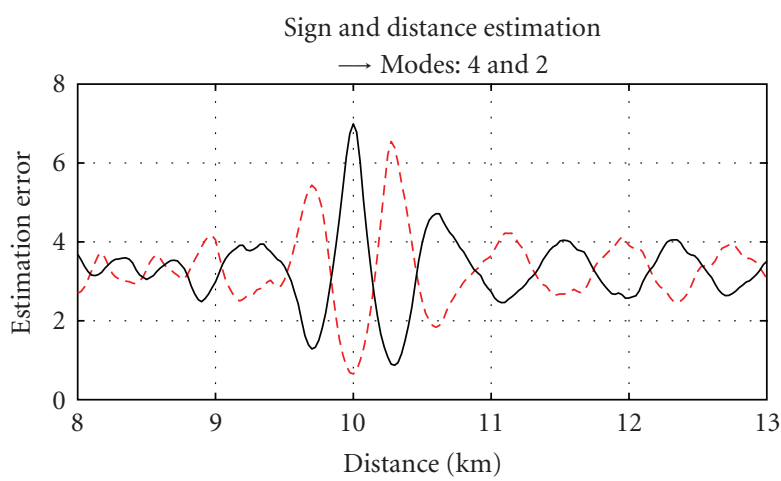

(c)

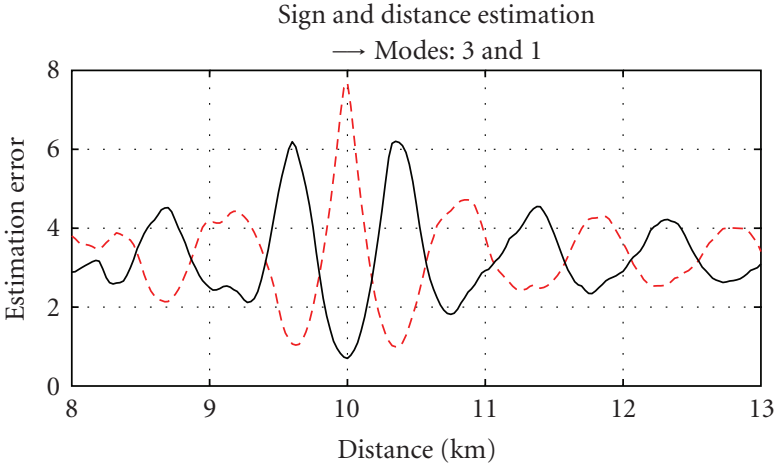

(b)

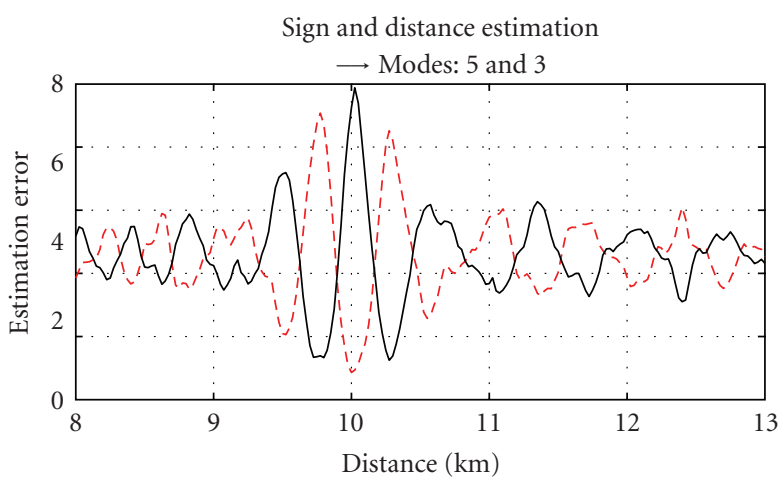

(d)

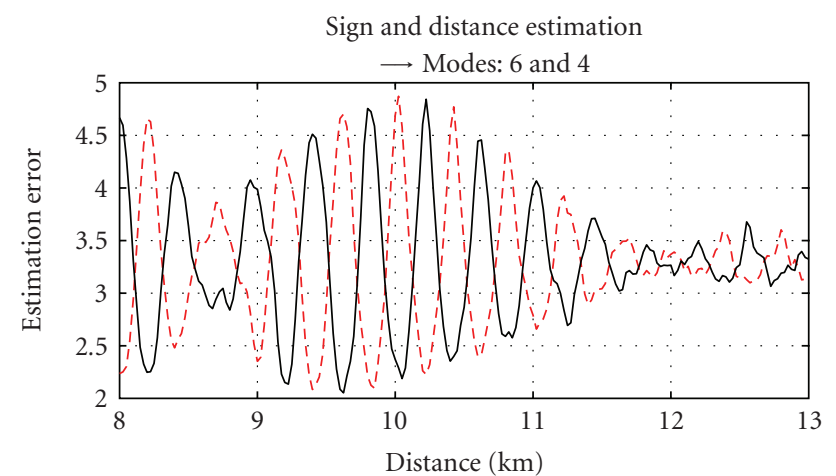

(e)

Figure 15: Cost functions $C$ of sign-distance estimator in a single hydrophone scenario. The results are given for mode couples: $(2,1),(3,1)$, $(4,2),(5,3)$, and $(6,4)$ and radial distance search zone $r \in(8,13) \mathrm{km}$. The source is $10 \mathrm{~km}$ distant from the hydrophone. Solid and dashed lines are given for two possible values of $\widehat{\Delta \phi}\left(m, n, z_{s}\right): 0$ and $\pi$.

1 to 6 is correct and the absolute signs are $1 \rightarrow+, 2 \rightarrow-$, $3 \rightarrow+, 4 \rightarrow+, 5 \rightarrow-$, and $6 \rightarrow+$.

6.1.2. Depth. For depth localization an estimation of mode excitation factors is needed. First, a modal filtering is performed on real and simulated data by $F T_{a}$ approach, and then for each analyzed mode, its mode excitation factor modulus is calculated as a mean over frequency. Moreover, mode signs estimated above can be used in the contrast function $G$.

In Figure 16 we show the result of depth localization. The performance is given for the methods "without mode signs" and "with mode signs." The difference in performance between two methods is relevant which results from the importance of taking into account mode signs when calculating the contrast function $G$. The method "with mode signs" eliminates the "mirror solutions" (decrease of the contrast function $G$ from $22.5 \mathrm{~dB}$ to $4.5 \mathrm{~dB}$ for a secondary peak at $105 \mathrm{~m}$ by adding mode signs). Figure 17 highlights also a problem that can appear when adding mode signs: if the mode sign estimation is false (here, sign of mode 3 is false), then the localization performance decreases significantly. However, as one can choose between 3 options when estimating mode signs (the current mode sign $n$ is 


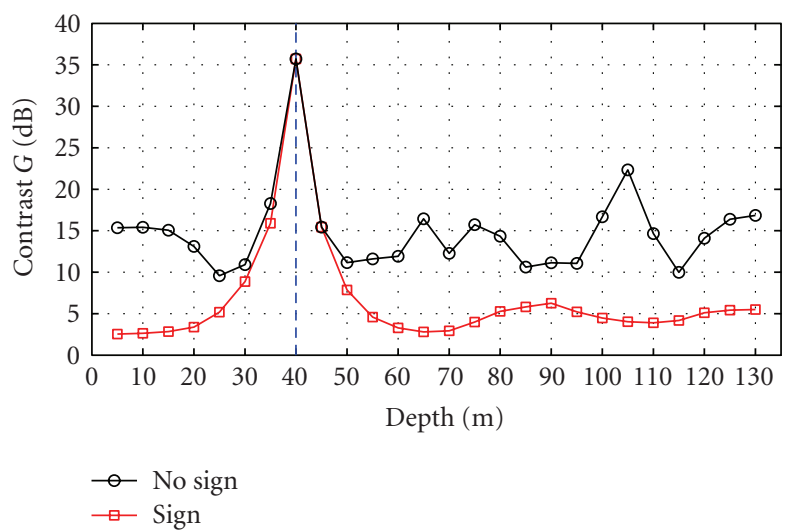

Figure 16: Contrast functions $G$ for depth source localization by $F T_{a}$ approach. The results are given for methods: without mode signs (circles) and with mode signs (squares). The source is located at $40 \mathrm{~m}$ of depth. The vertical resolution is $5 \mathrm{~m}$.

estimated using three inferior modes $m=\{n-3, n-2, n-$ $1\}$ ), the probability of this error should decrease (if the signal-to-noise ratio is sufficiently high).

Due to the oscillating character of modal functions and because we consider only modal function modulus, there exist "mirror" depths which give secondary peaks in the contrast function $G$ (Figure 14(a)). To explain this fact and the reason why they disappear when integrating mode signs in the depth estimation, let us present an example. Figure 18 shows 6 mode excitation factors extracted from simulated data at two different source depths: $40 \mathrm{~m}$ and $105 \mathrm{~m}$. For these two depths the mode excitation factor moduli are almost the same (Figure 18(a)), and so the difference in the contrast function for these depths is not relevant. When the complete information about mode excitation factors (i.e., modulus and sign) is considered (Figure 18(b)), these depths become discriminated. This is due to especially modes no. 2 , 6, but also 4 .

In the mono-dimensional configuration in lower signalto-noise ratio conditions the mode sign and distance estimations can be inaccurate. Also, the depth localization performance cannot be satisfied. Therefore, we propose the multi-dimensional configuration that is more robust and efficient due to a richer information about the source and the environment recorded on the HLA.

6.2. Horizontal Line Array. This section presents results of localization in distance and in depth using $F-K$ approach. The objective is to show the performance of $F-K$ localization method. The methods are validated in the environment and configuration described in Section 3 for a signal-to-noise ratio of $5 \mathrm{~dB}$. The distance between the source and the first hydrophone of HLA is $10 \mathrm{~km}$. The source depth is $105 \mathrm{~m}$ and the HLA is on the seabed.

According to the Shannon theorem and for the ULF band $\left(f_{\max }=100 \mathrm{~Hz}\right)$ the maximal spatial sampling should be done every $7.5 \mathrm{~m}$. Thus, in theory we could consider every second HLA hydrophone without any information lost (as the whole HLA samples linearly the space every

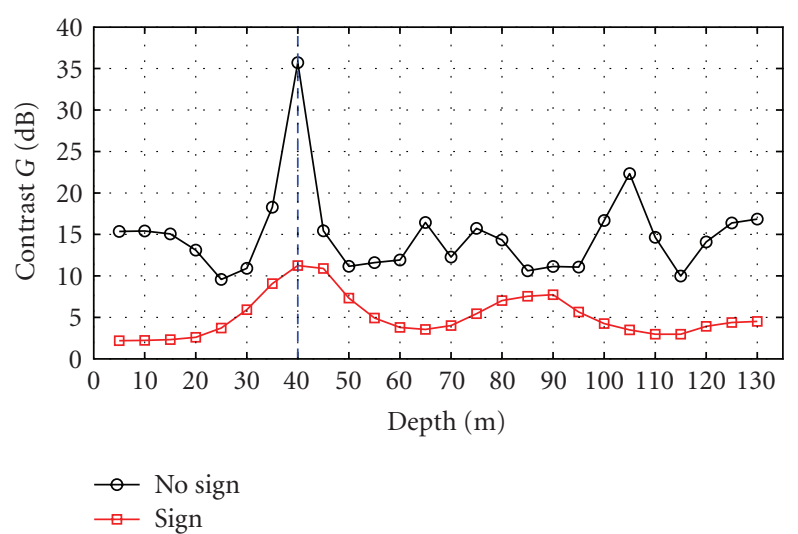

Figure 17: Contrast functions $G$ for depth source localization by $F T_{a}$ approach with false mode sign estimation. The mode sign no. 3 is estimated with an error (estimated as negative, but should be positive). The configuration localization is the same as in Figure 16.

TABLE 3: Results of the source distance estimation for $F T_{a}$ approach.

\begin{tabular}{lc}
\hline Mode couple & Estimated distance $(\mathrm{km})$ \\
\hline 2 and 1 & 9.925 \\
3 and 1 & 10 \\
4 and 2 & 10 \\
5 and 3 & 10 \\
6 and 4 & 9.625 \\
\hline
\end{tabular}

$3.347 \mathrm{~m}$ ). However, with a higher space sampling, better noise canceling algorithms can be implemented. What is more important, is a length of the HLA. When the length of HLA reduces, the localization performance decreases. This is provoked by a spreading of the signal in the $f-k$ plane which results from a not sufficiently long radial distance sampling of the modal signal [16]. Different issues of the use of HLA are discussed in [10].

The first step of the method is a $V_{\text {ref }}$ velocity correction which is done with the minimum value of the sound speed profile in water $V_{\min }=1508.4 \mathrm{~m} / \mathrm{s}$. Then, the $F-K$ transform is calculated and this representation is used for mode filtering. These modes are then analyzed for distance and depth estimations.

6.2.1. Distance. After $F-K$ filtering, the phase of each mode is calculated through a Fourier transform. The wavenumbers needed by the estimator defined in (19) are provided by Moctesuma simulator. This estimation is applied to each hydrophone of the HLA (240 estimations) [16].

We apply the estimator on five different mode couples: $(2,1),(4,1),(4,2),(5,2),(6,4)$, and $(7,4)$, and research erea $r \in(8,13) \mathrm{km}$ with step $\Delta r=25 \mathrm{~m}$. The estimated distance values are given in Figure 19 and its mean values are given in Table 4. Moreover, the sign-distance estimator gives as a result mode sign. In multi-dimensional case, we dispose of $N_{r}$ estimations of mode signs for each mode couple option. For a mode number equal to 7 , the sign-distance estimator is applied on following mode couples: $(2,1)$ (for mode 2), 


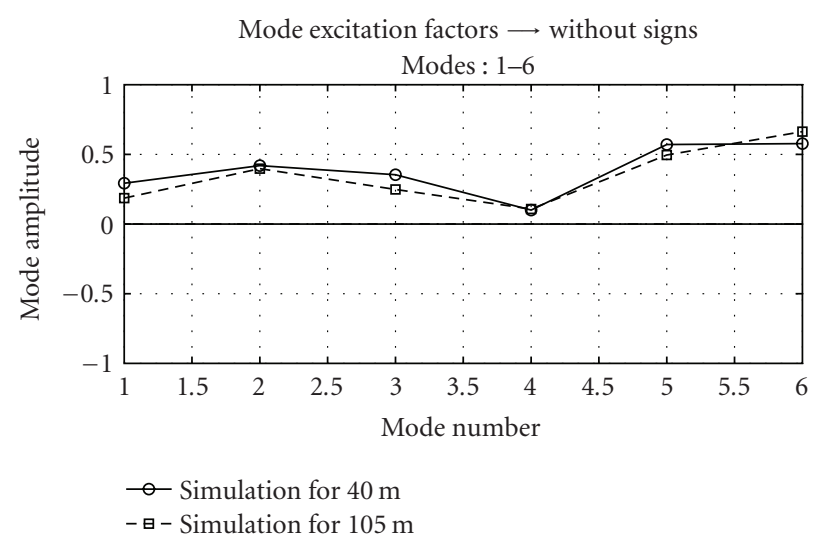

(a)

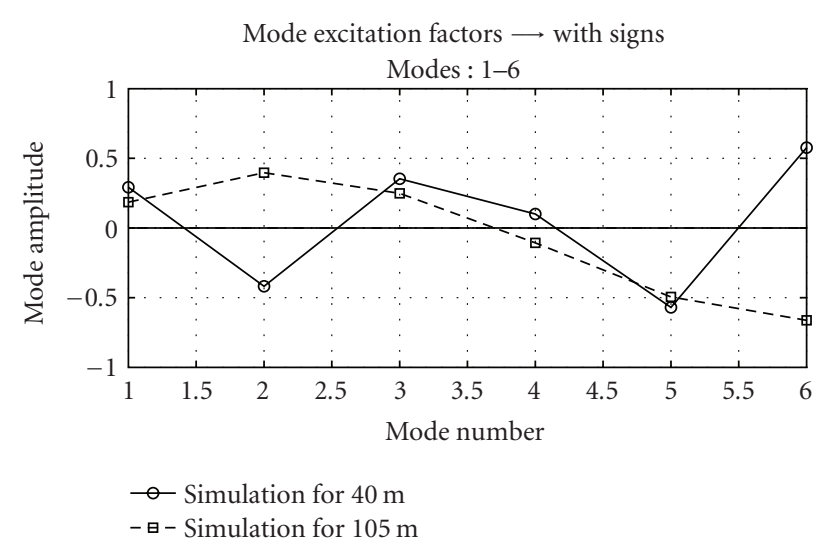

(b)

Figure 18: Mode excitation factors extracted from 2 simulations: source at $40 \mathrm{~m}$ and $105 \mathrm{~m}$ of depth. (a) represents the mode excitation factor modulus (no sign information) and (b) shows the mode excitation factor modulus combined with sign information.

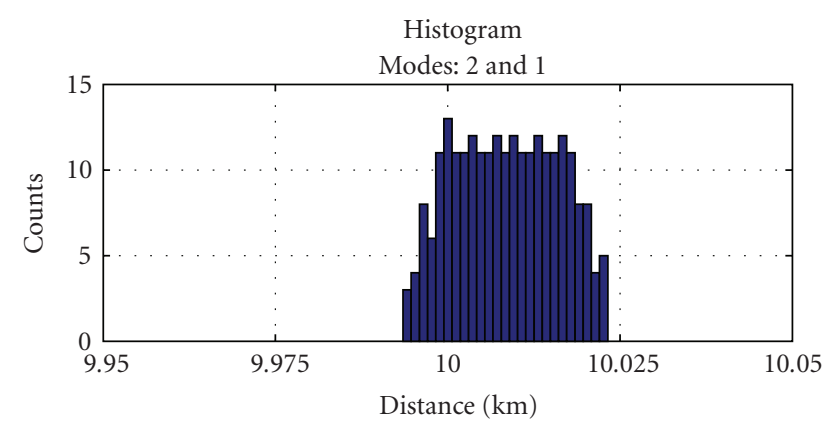

(a)

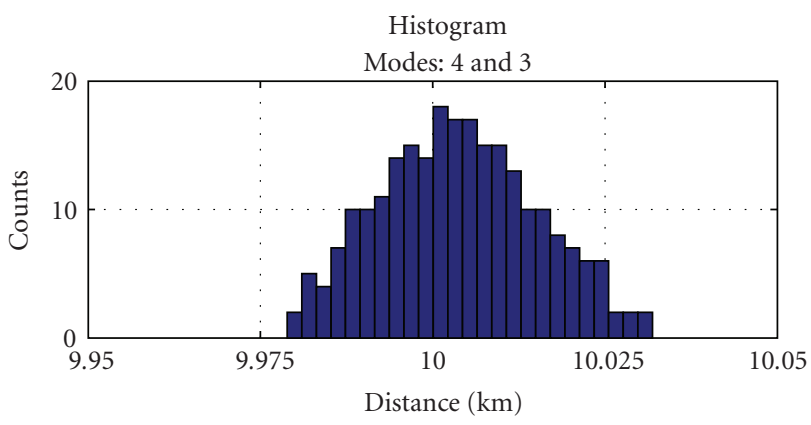

(c)

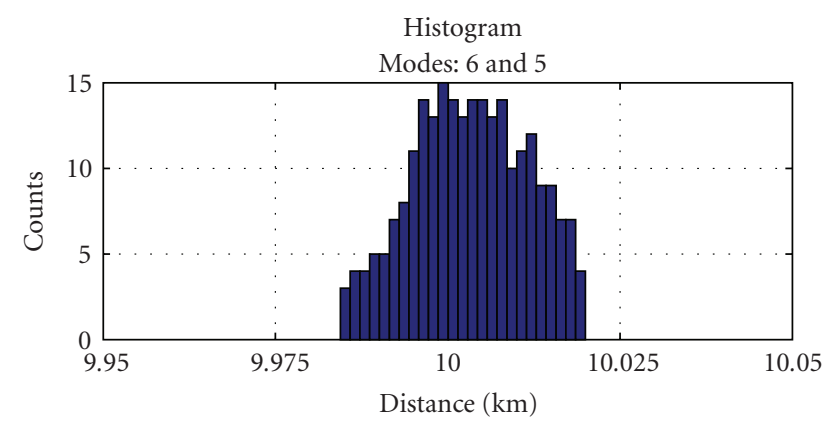

(e)

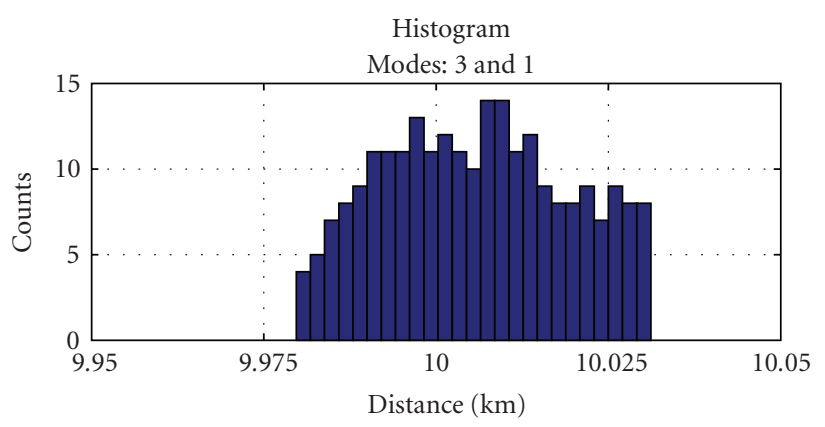

(b)

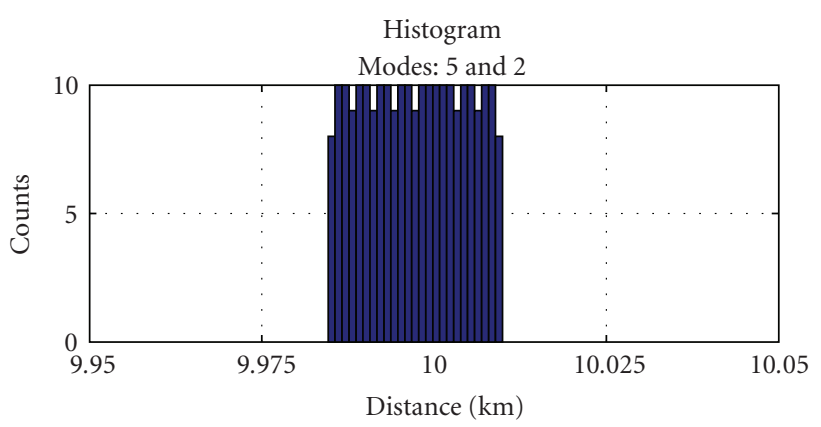

(d)

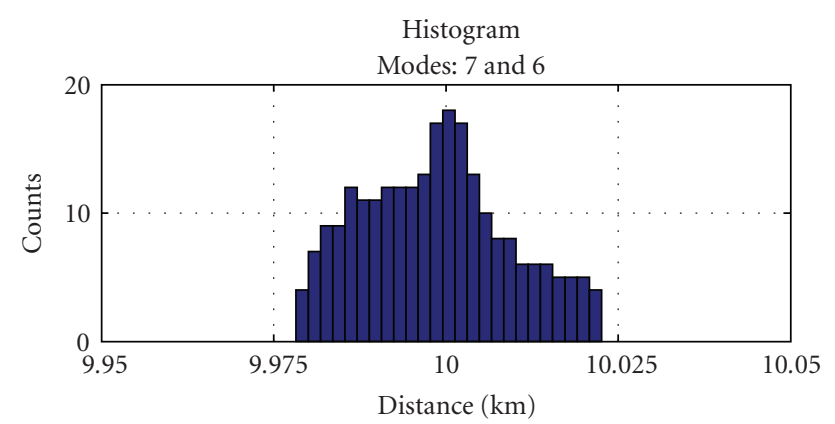

(f)

FIgURE 19: Results of distance estimation by F-K method for 6 mode couples. For each mode couple the estimation is done for all HLA hydrophones (240 distance estimations). The true value of distance is $10 \mathrm{~km}$. 
TABLE 4: Mean values of source distance estimation for multidimensional approach. The bin width is $5 \mathrm{~m}$.

\begin{tabular}{lc}
\hline Mode couple & Mean distance value $(\mathrm{km})$ \\
\hline 2 and 1 & 10.01 \\
3 and 1 & 10.01 \\
4 and 3 & 10.005 \\
5 and 2 & 10 \\
6 and 5 & 10.005 \\
7 and 6 & 10.005 \\
\hline
\end{tabular}

$(3,1),(3,2)$ (for mode 3$),(4,1),(4,2),(4,3)$ (for mode 4$)$, $(5,2),(5,3),(5,4)$ (for mode 5), $(6,3),(6,4),(6,5)$ (for mode $6),(7,4),(7,5)$, and $(7,6)$ (for mode 7$)$ and the user has to select the couple he wants to use. This information is used here to maximize the probability of correct choice within available options for each estimation step. As the mode sign estimation is sequential it is primordial to not commit an error at the beginning to avoid its propagation. At each step (for each mode sign estimation) a series of 3 parameters is calculated to help the user in taking the decision. For the first step these parameters are calculated once (for the couple $(2,1))$, for the second step we dispose of two set of parameters (for the couples $(3,1)$ and $(3,2)$ ), and for the following steps we have always three sets of parameters. These parameters are defined as follows.

(i) Choice reliability:

$$
\frac{\mid N-\text { floor }\left(N_{r} / 2+1\right) \mid}{\text { floor }\left(N_{r} / 2+1\right)} * 100,
$$

where $N$ is a number of sign changes $\left(N \in\left[0, N_{r}\right]\right)$. This criteria should be maximal.

(ii) Estimation variability:

$$
\left|\sum_{N_{r}}\left(\mathbf{r}_{e}\right)^{\prime \prime}\right|^{2},
$$

where $\mathbf{r}_{e}$ denotes a set of $N_{r}$ distance estimations and " denotes a second derivative with respect to the hydrophone number. For the no-error estimation of distance the first derivative is equal to interhydrophone distance. Then, the second derivative is equal to zero as the first derivative is a constant function. This criterion allows to measure the variability of distance estimations across all hydrophones and should be minimal.

(iii) Error distance estimation:

$$
\frac{\left|1 / N_{r} \sum_{n=1}^{N_{r}}\left(r_{e}^{n, i+1}\right)-r_{e}^{i}\right|}{r_{e}^{i}},
$$

where $r_{e}^{n, i+1}$ denotes a distance estimation for $n$th hydrophone at actual step analysis $(i+1)$ and $r_{e}^{i}$ denotes the final estimation of distance from previous step (i). This criterion allows cancel secondary peak solutions for which the first two criteria gave good results and should be obviously minimal.

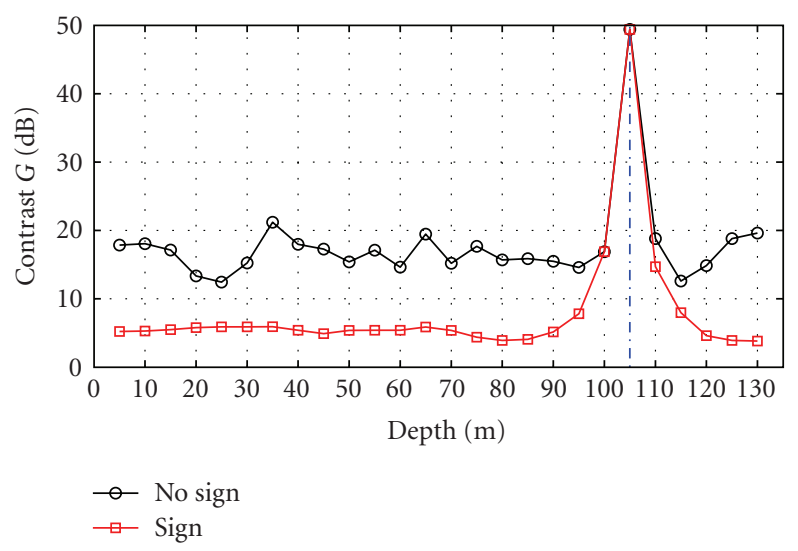

Figure 20: Contrast functions $G$ for depth source localization by $F-K$ approach. The results are given for methods: without mode signs (circles) and with mode signs (squares). The source is located at $105 \mathrm{~m}$ of depth. The vertical resolution is $5 \mathrm{~m}$.

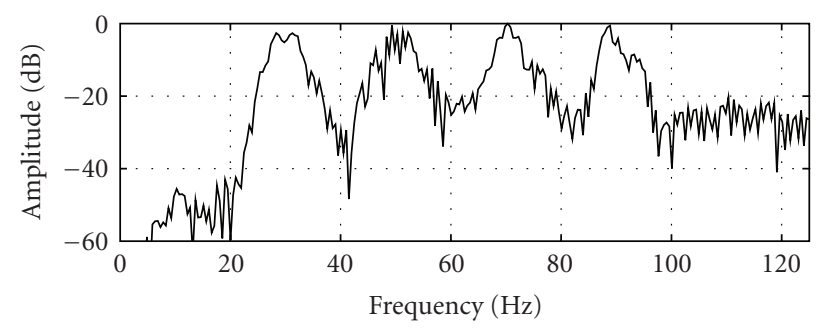

FIgURE 21: Result of the source spectrum estimation for source ULF-2 (to be compared with Figure 4(d)).

For the example presented here, the mode signs were estimated on the same mode couples as distance. The estimation of signs of modes no. 1 to 7 is correct and the absolute signs are $1 \rightarrow+, 2 \rightarrow+, 3 \rightarrow+, 4 \rightarrow-, 5 \rightarrow-$, $6 \rightarrow-$, and $7 \rightarrow-$.

6.2.2. Depth. After modal filtering, the mode excitation factor modulus of each mode is calculated as a mean over the $f-k$ region. Moreover, the sign-distance estimator can be used for mode signs estimation.

In Figure 20 we show the result of depth localization. The performance is given for method "without mode signs" and "with mode signs." The difference between both methods is relevant which confirms the importance of taking into account mode signs when calculating the contrast function $G$. The method "with mode signs" eliminates the "mirror solutions" (decrease of the contrast function from $21 \mathrm{~dB}$ to $6 \mathrm{~dB}$ for a secondary peak at $35 \mathrm{~m}$ by adding mode signs).

6.3. Source Spectrum Issue. In Section 5.3 we described a simple method of estimation of the source spectrum. Now, we quantify the impact of this estimation on depth localization.

Let us consider an example of depth localization in the environment described in Section 3. The source ULF-2 is 


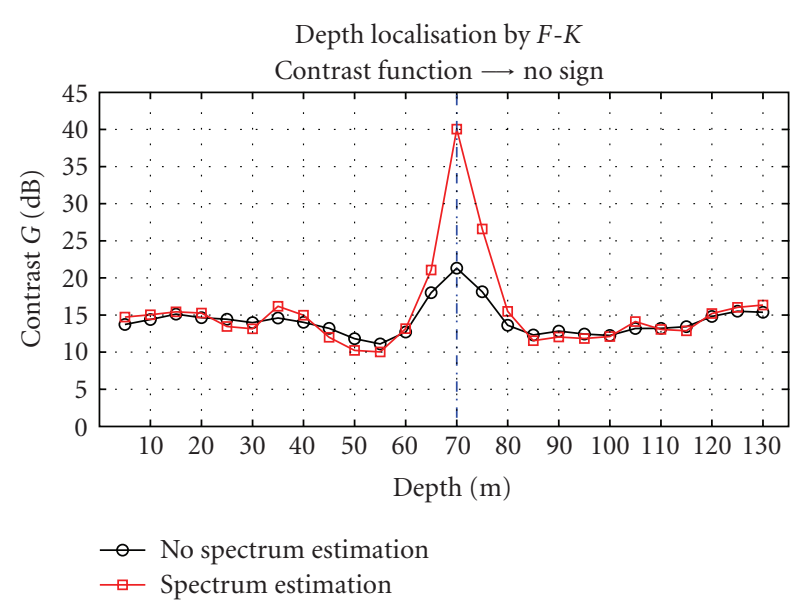

(a)

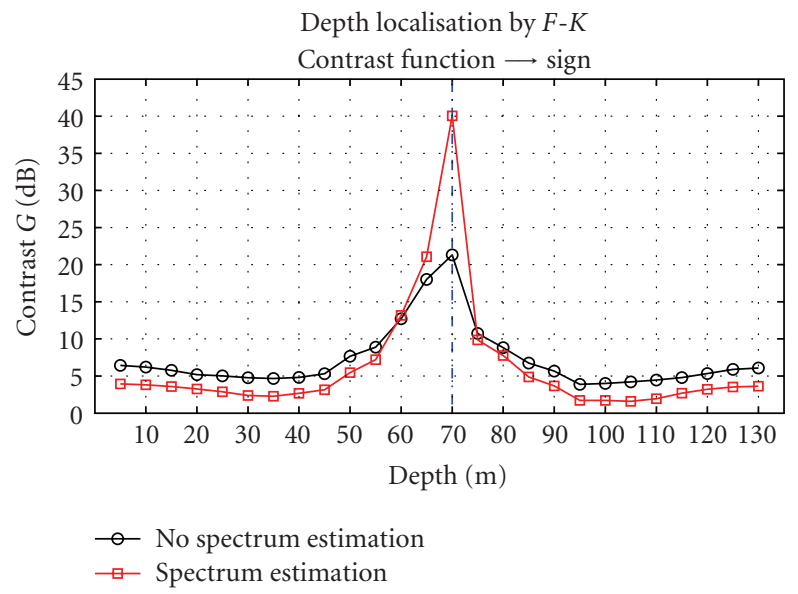

(b)

FIGURE 22: Contrast functions $G$ for depth source localization by $F-K$ approach combined with source spectrum estimation. The simulation is given for source ULF-2 located at $70 \mathrm{~m}$ of depth and $10 \mathrm{~km}$ distant from the HLA. (a) shows the gain given by taking into account the estimated spectrum of an unknown source for localization method without mode signs. (b) shows this gain in case of localization algorithm with integrated mode signs into the contrast function.

located at $70 \mathrm{~m}$ of depth. As we do not know the spectral properties of the localized source we consider two cases.

(i) We use a source with flat spectrum for simulation of the replica field (source ULF-1)-common approach when unknown source.

(ii) We estimate a source spectrum by the method defined in (29) and use it to simulate the replica field.

In Figure 21, we present spectrum of the source ULF2 estimated by the proposed method (compare with Figure $4(\mathrm{~d})$ ). The results of localization without and with source spectrum estimation are shown in Figure 22. We can note that estimating the source spectrum improves considerably the localization performance (of about $20 \mathrm{~dB}$ in the example).
Nevertheless, our method is designed for broadband sources. Therefore, even if spectral characteristics of the source are perfectly known, but present narrowband or comb-type structures, the localization performance decreases. The performance decrease due to nonbroadband source is higher than the gain due to acquaintance of source spectral characteristics.

\section{Conclusion}

In this paper we propose passive source localization in shallow water based on modal filtering and features extraction. The depth and distance of an Ultra Low Frequency source are estimated in the mono-dimensional configuration (a single hydrophone) and in the multi-dimensional configuration (a horizontal line array). The localization techniques are, respectively, based on adapted Fourier transform and frequency-wavenumber transform. In both representations modes are separable and thus can be filtered. We discuss modal filtering tools, then the localization itself is performed.

For distance estimation, we base our localization method on the analysis of mode phases. The proposed distance estimator is naturally combined with mode sign estimator. For depth localization, we use matched-mode processing, a technique that widely demonstrated its performance in a shallow water environment. The principle is based on comparison (by a contrast function) of mode excitation factors extracted from real data with a set of mode excitation factors (for simulated source depths) extracted from replica data (modeled with Moctesuma). We demonstrate that adding the mode signs to the mode excitation factor modulus improves significantly the localization performance in depth. We also propose a method of estimation of the source spectrum, which is very important for depth localization using Matched-Mode Processing.

The localization results, in depth and distance, obtained on signals simulated with Moctesuma in realistic geophysical conditions are very satisfactory and demonstrate the performance of the proposed methods.

\section{Acknowledgment}

This work was supported by Project REI 07.34.026 from the Mission pour la Recherche et l'Innovation Scientifique (MRIS) of the Delegation Generale pour l'Armement (DGAFrench Departement of Defense).

\section{References}

[1] F. B. Jensen, W. A. Kuperman, M. B. Porter, and H. Schmidt, Computational Ocean Acoustics, AIP Press, New York, NY, USA, 1994.

[2] A. B. Baggeroer, W. A. Kuperman, and P. N. Mikhalevsky, "An overview of matched field methods in ocean acoustics," IEEE Journal of Oceanic Engineering, vol. 18, no. 4, pp. 401-424, 1993.

[3] J. A. Fawcett, M. L. Yeremy, and N. R. Chapman, "Matchedfield source localization in a range-dependent environment," The Journal of the Acoustical Society of America, vol. 99, no. 1, pp. 272-282, 1996. 
[4] G. R. Wilson, R. A. Koch, and P. J. Vidmar, "Matched-mode localization," The Journal of the Acoustical Society of America, vol. 104, no. 1, pp. 156-162, 1998.

[5] E. C. Shang, C. S. Clay, and Y. Y. Wang, "Passive harmonic source ranging in waveguides by using mode filter," The Journal of the Acoustical Society of America, vol. 78, no. 1, pp. 172-175, 1985.

[6] N. E. Collison and S. E. Dosso, "Regularized matchedmode processing for source localization," The Journal of the Acoustical Society of America, vol. 107, no. 6, pp. 3089-3100, 2000.

[7] C. W. Bogart and T. C. Yang, "Comparative performance of matched-mode and matched-field localization in a rangedependent environment," The Journal of the Acoustical Society of America, vol. 92, no. 4, pp. 2051-2068, 1992.

[8] W. A. Kuperman, W. S. Hodgkiss, and H. C. Song, "Phase conjugation in the ocean: experimental demonstration of an acoustic time-reversal mirror," The Journal of the Acoustical Society of America, vol. 103, no. 1, pp. 25-40, 1998.

[9] C. Prada, J. de Rosny, D. Clorennec, et al., "Experimental detection and focusing in shallow water by decomposition of the time reversal operator," The Journal of the Acoustical Society of America, vol. 122, no. 2, pp. 761-768, 2007.

[10] C. W. Bogart and T. C. Yang, "Source localization with horizontal arrays in shallow water: spatial sampling and effective aperture," The Journal of the Acoustical Society of America, vol. 96, no. 3, pp. 1677-1686, 1994.

[11] B. Nicolas, J. I. Mars, and J.-L. Lacoume, "Source depth estimation using a horizontal array by matched-mode processing in the frequency-wavenumber domain," EURASIP Journal on Applied Signal Processing, vol. 2006, Article ID 65901, 16 pages, 2006.

[12] G. Le Touzé, B. Nicolas, J. Mars, and J.-L. Lacoume, "Matched representations and filters for guided waves," IEEE Signal Processing Letters. In press.

[13] R. G. Baraniuk and D L. Jones, "Unitary equivalence: new twist on signal processing," IEEE Transactions on Signal Processing, vol. 43, no. 10, pp. 2269-2282, 1995.

[14] T. C. Yang, "A method of range and depth estimation by modal decomposition," The Journal of the Acoustical Society of America, vol. 82, no. 5, pp. 1736-1745, 1987.

[15] X. Cristol, J.-M. Passerieux, and D. Fattaccioli, "Modal representations of transient sound pulses in deep and shallow environments, with investigations about detailed space and time correlation of propagated waves," MAST, 2006.

[16] M. Lopatka, B. Nicolas, G. Le Touzé, et al., "Robust underwater localization of ultra low frequency sources in operational context," in Proceedings of the Uncertainty Analysis in Modelling (UAM '09), Nafplion, Greece, 2009.

[17] G. Le Touzé, B. Nicolas, J. I. Mars, and J.-L. Lacoume, “Timefrequency representations matched to guided waves," in Proceedings of the IEEE International Conference on Acoustics, Speech and Signal Processing (ICASSP '06), vol. 3, pp. 440-443, 2006.

[18] A. Papandreou-Suppappola, R. Murray, B.-G. Iem, and G. F. Boudreaux-Bartels, "Group delay shift covariant quadratic time-frequency representations," IEEE Transactions on Signal Processing, vol. 49, no. 11, pp. 2549-2564, 2001.

[19] M. McClure and L. Carin, "Matching pursuits with a wavebased dictionary," IEEE Transactions on Signal Processing, vol. 45, no. 12, pp. 2912-2927, 1997.

[20] G. Le Touzé, localisation de source par petits fonds en UBF $(1-100 \mathrm{~Hz})$ à l'aide d'outils temps-frequence, Ph.D. dissertation, INP, Grenoble, France, 2007.
[21] B. Nicolas, G. Le Touzé, and J. I. Mars, "Mode sign estimation to improve source depth estimation," in Proceedings of the IEEE International Conference on Acoustics, Speech and Signal Processing (ICASSP '08), pp. 2437-2440, Las Vegas, Nev, USA, 2008.

[22] S. M. Jesus, "Normal-mode matching localization in shallow water: environmental and system effects," The Journal of the Acoustical Society of America, vol. 90, no. 4, pp. 2034-2041, 1991.

[23] J. H. Wilson, S. D. Rajan, and J. M. Null, "Inversion techniques and the variability of sound propagation in shallow water," IEEE Journal of Oceanic Engineering, vol. 21, no. 4, p. 321, 1996.

[24] B. Nicolas, J. Mars, and J. L. Lacoume, "Geoacoustical parameters estimation with impulsive and boat-noise sources," IEEE Journal of Oceanic Engineering, vol. 28, no. 3, pp. 494-501, 2003.

[25] G. Le Touzé, J. I. Mars, and J.-L. Lacoume, "Matched timefrequency representations and warping operator for modal filtering," in Proceedings of the European Signal Processing Conference (EUSIPCO '06), Florence, Italy, September 2006. 